\title{
QUELQUES OBSERVATIONS SUR LE TRACÉ ET LA PARTITION DE LA BANDE A RELIEFS HISTORIES DE LA COLONNE TRAJANE
}

Radu Florescu

1.1. La Colonne Trajanne a constitué, depuis assez longtemps, une préoccupation sinon constante du moins réiterée périodiquement dans le milieu des historiens et des historiens d'art de la Rome antique. Les dernières travaux la concernant: celui de Frank Lepper et Sheppard Frere ${ }^{1}$ et l'autre du aux quatre savants italiens - Settis, La Regina, Agosti et Farinella ${ }^{2}$ - offrent une reproduction complète des reliefs et, la première une étude archéologique pas seulement de la Colonne mais aussi des autres témoignages, monumentaux ou écrits, corélatifs, comme aussi, du point de vue téchnique, le marquage, sur les reproductions des reliefs, de quatre axes des fenêtres de l'éscalier en colimaçon, tandis que la seconde pose, pour la premiére fois dépuis Cichorius ${ }^{3}$ et Lehmann-Hartleben ${ }^{4}$, le problème de la composition de la bande à reliefs. Deux principes, surtout, formulés dans cette dernière ordre d'idées, sont à retenir: celui d'une syntaxe figurale plus complèxe et mieux articulée que la simple enfilade - scène aprés scène - proposée par Cichorius et celui d'un rhytme compositionel générateur d'une correspondance sur la verticale de scènes similaires. Dans cette ordre d'idées il ne faut pas ommettre les interventions d'Allain Mallissard ${ }^{5}$, qui en fondamentant le concèpt de «analyse filmique» du relief de la Colonne a ouvert des nouves perspéctives à la restitution de la structure figurale-narative et par ça, à la compréhension de ceci. Tous ces idées, bien intéressantes et ouvrant des réelles pérspectives pour l'étude plus approffondie du relief continuu de la Gōlonne, sont quand-même restées dans l'état d'énnonce théorique et personne n'a guerre soulevé le problème téchnique du traçage et de la partition de la bande figurée.

Une hypothèse de travail généralement accéptée est celle du Maestro, c'est à dire de la grande personalité artistique qui a conçue la frise et qui probablement l'a - téchniquement parlant projétée et a surveilé et dirigé l'éxécution. Cest aussi une des prémisses des considérations suivantes. Parmis celles-ci on doit mentionner l'éxistence d'un support géométrique du traçage et de la partition, c'est à dire d'un traçé régulateur s'appuyant sur des particularitées du fût de la Colonne, comme aussi la qualité de ce tracé de pouvoir être utilisé à l'aide d'un assez simple appareil de mensuration - le plus probable une corde à noeuds.

n faut, semblablement, faire «la part du diable » c'est à dire prendre conscience des handlcaps parsémant la voie de tout essaye de restitution du trayail de concéption et de projéction perpétré par le Maestro. Dans cette pérspéctive on doit d'abord tenir compte de tolérances de l'éxécution du relief. Quelques-unes de ces tolérances se justifient par des raisons artistiques comme

\footnotetext{
${ }^{1}$ Lepper Frank, Frere Sheppard. Trajan's Column. Gloucester; Allan Sutton; 1988; 340 p.;1 h.;CXII pl.

2 Settis Salvatore, La Regina Adriano, Agosti Giovanni, Farinella Vincenzo. La Colonna Traiana. Torino, Einaudi, 1988; 600 p., 288 pl.; 92 fig.

${ }^{3}$ Cechorius Conrad. die Reliefs der Traiansstule, vol. II, Berlin, Walter de Gruyter, 1896; vol, III, Berlin, Walter de Gruyter, 1900.

${ }_{4}^{4}$ Lehmann-Hartleben Karl. Die Traianssäule: ein rómische Kunstwerk zu Beginn der Spätantike. 2 vol, Berlin-Leipzig, Walter de Gnyter, 1926

${ }^{5}$ Mallissard Allain.Pour une étude filmique de la Colonne Trajane. Dans "Actes du IXe Congrès International d'études sur les frontières romaines" Mamaia, 6 - 13 septembre 1972, Editura Academiei, Bucureşti/Böhlau Verlag, Köln-Wien,1974, p. 545 550; Idem. La comparaison avec le cinéma permet-elle de mieux comprendre la firise continue dela Colonne Trajane ?" Röm. Mitt. LXXXIII (1976).
} 
par exemple les «liaisons» qui assurent le «coulage» continuel de scènes l'une de l'autre. D'autres sont dues peut-être à des erreures locales. De toute manière, ainsi qu'on va voir plus loin, les tollérances sont contenue dans des limites convénables. Il est nécessaire de compter aussi avec le fait que le traçé régulateur comme aussi les partitions ont étě mésurées sur le fût pas encore amminci par la sculpture figurale. On peut éssayer de compensen la difference de rayon, en général, mais il n'est pas possible de calculer la difference entre toutes les dimensions originaires et celles prélevées sur les reliefs dans leur êtat actuel. Quand-même ces diffèrences aussi ne sont plus importantes que de quelques millimètres.

En fin il est necessaire de prendre en considération les circonstances concrètes de ce travail de remodélage: toutes les mensurations ont été faites sur les copies des reliefs existantes dans le Muzeul Naţional de Istorie a României de Bucarest; leurs dimensions ne sont pas tout-à-fait identiques aux celles de la Colonne elle-même; en effet; le materiel - ciment armé à péllicule de gypse melé à de la poudre de marbre - a été soumis à des contractions au moment de la « prise ». Le coéficient de contraction est sans doute petit, mais il n'est pas sûre qu'il a été homogène et il ne peut pas être calculé qu'avec incértitude.

1.2 La structure constructive même de la Colonne offre un très tentant modèle de traçé regulateur. En effet, les 18 tambours (celui avec le tor de base y compris) avec leurs fugues intermédiaires combinées avec les quatre files de fenêtres assurent la trame orthogonale de base. Cette trame a la propriétée d'être équidistante : la hauteur moyenne des tambours est sensiblement égale à la huitième part de la circumfèrence moyenne. Toutes les deux mésurent en moyenne 1,48 m. c'est à dire 5 pieds romains. Il semble donc que la solution soit simple et aisée, contenue pour ainsi dire dans les dates fondamentales de la construction. Il y a quand-même quelques détails qui viennent compliquer suffisament la situation: d'abord l'hauteur des tambours n'est pas tout à fait constante, notamment elle varie, sans aucune règle, entre $1,4785 \mathrm{~m}$. et $1,554071875 \mathrm{~m}$. ${ }^{6}$.A son tour le fût n'est pas cylindrique mais tronconique, donc la longueur du cercle varie - probablement d'une manière régulière - de $10,3306 \mathrm{~m}$. au sommet à $11,5552 \mathrm{~m}$. (à peu près 40 pieds romains) à la base'. Il s'en suit avec evidence que tout traçé regulateur devait être ajustable aux variations des dimensions de base, c'est à dire d'avolf une structure modulaire, repportable donc à une seule dimension de base, choisie à l'interieur du monument - un module. Accéptons comme hypothèse de travail fondamentale que ce module est un quadrilatère dont l'hauteur est égale à celle du tambour et la largeur est la huitième partie de la circumfèrence. Thèoriquement les deux grandeurs sont égales et le module ainsi chaisi est un carré. La division de toute la surface de la Colonne en des carreaux de $5 \times 5$ pieds romains en partant de la grande trame marquée par les fugues des tambours et par les files de fenêtres était facilement réalisable avec une simple corde en tendant celle-ci sur l'interaxe des deux fenêtres voisines et en divisant la grandeur resultée par deux et par quatre. Mais la partition n'était pas si simple à obtenir si on voulait utiliser les unitées du système du pied romain. La seconde hypothèse de travail sérait-ce, donc, que le Maestro a rennoncé à atiliser la règle gradée pour se fier éxclusivement à la corde et aux coordonées inscrites dans la construction même du monument. Il est probable qu'il y avait une liaison, plus probablement operationelle, entre les 8 verticales du tracé

\footnotetext{
${ }^{6} \mathrm{v}$. Tabelle $\mathrm{nr} .1$ et 2 , cf.

${ }^{7}$ v. Tabelle nr. 3, cf. Forescu Forea Bobu. Die Trajanssäule, Bucureşti-Bonn, Akademie-Rudolph Habelt Verlag, 1969, p.39, Tabelle 1, pl.15.
} 
regulateur et les canelures qui aparaissent à la base du capitel et dont quatre correspondent aux axes des fenêtres ${ }^{8}$.

Il est possible, donc, d'imaginer le fût de la Colonne pas encore degrossi avec les coordonnées principales - les fugues des tambours et les files des fenêtres - déjà mises en places. Le tracé regulateur ainsi constitué consistait en une reseau de $8 \times 18$ carreaux majeurs- les modules divisés chacun en $16 \times 16$ petits carreaux - les modules de base. Si, en principe, chaque module mésurait 5 pieds romains (1 pas romain) de coté, chaque module de base mésurait 5 pouces (digit) de coté. Les tollerances en hauteur et les réductions proportionnelles des diamètres étaient compensées automatiquement dés par le trasage originaire du réseau. Sur cette trame était suffisant de tracer une ligme oblique dont les interséctions avec les coordonées magistrales du tracé regulateur étaient déterminées découlant diréctement des raports proportionels entre le tracé - réspectivement le fût de la Colonne - et la bande spirale. Celle-ci se déroulait sur 23 spires ${ }^{9}$ dont les deux éxtrèmes en forme de triangle, c'est-à-dire ayant une seule coté oblique, l'autre étant horizontale, pour s'inscrire dans les limites du fût de la Colonne. L'hauteur moyenne de la bande figurée était de $1,11 \mathrm{~m} .{ }^{10}$ très proche de celle de $3 / 4$ de l'hauteur du tambour. Cette proportion de $3 / 4$ ne serait-ce pas la proportlon ihitiale, établie fonction du nombre des tambours et de la qualité de columna centenaria attribué au monument ? En effet le rapport des 24 spire à 18 tambours est de $4 / 3$. Si on accepte que la partition initale a été ajustée pour assurer la place necessaire à la plinthe et au tor de la base de la Colonne (qui ensemble occupent tout juste les $3 / 4$ du premier tambour) à l'intérieur de 100 pieds romains cannoniques il nous en reste précisement l'espace nécessaire pour 23 spires. C'est une solution simple et aisée aussi pour la césure d'entre les deux guerres - le premier problème important de partition de la bande spirale - temandant le transfèr de cette cèsure de la fin dela 12-e spire au milieu de celle-ci même. On peut admettre que les modules de base étaient groupés par quatre, pour facilliter les mensurations operationelles au cours de l'éxecution. De même il faut penser que la grille orthogonale a été réalisé depuis le début de l'éxecution en réunissant, d'abord, les réperes des ordonnées - les milieux des canelures du capitel comptées de trois en trois depuis l'axe SE et les points correspondant aux axes et aux diagonales du socle (v. fig. 1) - et puis en les marquant sur chaque limite de tambour. Dahs une maniěne similaire unt ěté marquées les abscisses sur les quatre axes des fenêtres. Avec cet appareil rélativement simple a été tracé la ligne diagonale delimitant la bande figurée en prenant de grandeurs linéaires sur les axes et en les réunisant ( v. fig.2 - 6). Une analyse de la fig. 2 vaille une démonstration du mode d'opération probable. Ainsi le traçage de la ligne diagonale commence à l'intérsection de l'axe SE avec l'abscisse 0 . Le second point est déterminé par l'intersection de l'axe NE avec l'abscisse I (c'est à dire la fugue entre le tambours 1 et 2). Le point suivant peut être déterminé en prenant, en sens ascendant, trois $\boldsymbol{m b}$ (modules de base) sur l'axe NV en partant de l'intérsection de celle-ci avec l'abscisse I. Le troisième point s'obtient en prenant six $\boldsymbol{m} \boldsymbol{b}$ sur l'axe SV en partant de l'interséction avec l'abscisse 1.En fin, sur l'axe SE la spire finit à huit $\boldsymbol{m} \boldsymbol{b}$ de l'interséction avec l'abscisse I. La bande figurée n'as pas encore atteint sa hauteur normale.

Le plus facile mode de déterminer le point suivant de la bande spirale c'est de descendre 4 $\boldsymbol{m} \boldsymbol{b}$ sur l'axe NE, de l'interséction de celle-ci avec l'abscisse II. Le second point de la spire 2 coïncide avec l'interséction de l'axe NV avec la même abscisse. Le troisième peut être obtenu en montant sur

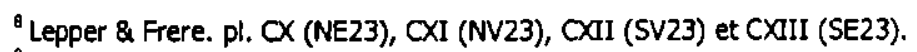

${ }^{9}$ Settis, La Regina, Agosti, Farinella, p.46

${ }^{10}$ Cette grandeur représente la moyenne de 12 mensurations dirèctes prises sur les reliefs de Bucharest.
} 
l'axe SV 2 mb de l'interséction de cette axe avec l'abscisse II. En fin; la seconde spire finit sur l'axe SE, $4 \boldsymbol{m} \boldsymbol{b}$ au dessus de l'interséction avec l'abscisse II.

En partant du principe que, pour des raisons opérationelles, le Maestro a indiqué toujours la distance de l'interséction la plus proche on peut dresser un tableau modèle des dimensions detèrminantes pour le tracée de la bande spirale (v:tableau 3). De ce tableau, comme aussi des diagrammes de la bande figurée se dégage une variation de la hauteur de la bande de $-1 /+3 m b$ c'est à dire de moins de 5\%, avec des fréquentes régularisations à $12 \mathrm{mb}$. On peut aussi discerner une ajustation drastique dans les trois dernières spires. Sans doute ces tollerances et ces ajustations témoignent une surveillance assez laxe de l'éxecution des reliefs, d'ailleurs bien en accord avec les possibilitées assez éxigues d'éxaıniner de près l'Oeuvre. En même temps le traçage de la bande spirale était la chose la plus simple et la plus facile de tout le procéssus de composition, ou mieux dit, de projection de l'histoire figurée des deux guerres daciques (tableau 4). Si au temps de Conrad Cichorlus il était possible de concevoir la bande figurée comme une succéssion d'instantanés pris sur le champs de bataille, tout à fait similaire à un réportage photographique de front, les recherches ultérieures tant de Adriano La Regina ${ }^{11}$ que de Lepper et Frere ${ }^{12}$ ont mis en valeur l'idée d'une composition bien complèxe, comportant un essaye de rendre un temps et un éspace variable dans les deux dimension du relief. Si on ajoute à ça l'analyse de spécial interêt proposée par Alain Malissard ${ }^{13}$ comme aussi l'hypothèse riche en conséquences de Gauer ${ }^{14}$ - même si on repousse la parfaite identitée de la bande figurée avec le texte de Trajan - il faut essayer d'abord de cerner les grandes articulations de la composition de l'histoire figurée, en établissant aussi les differents traitement des simultanéitées et des séquences et après ça seulement de préciser les scènes - avec leurs limites.

2.1. La syntaxe de la naration figurée - en tant que nous pouvons nous rendre compte de l'ordonnance des huits livres du De bello dacico de Trajan - éxigeait une césure majeure juste au. milieu de la bande figuré pour marquer la pause d'entre les deux guerres. En effet, la scéne LXXVIII (Victoire) est emplaçée au croisement de l'abscisse 9 avec l'ordonnée NV, c'est-à-dire au juste milieu dela bande dessinée. La moitiée antarieure de la bande devait contenir la première guerre dacique et celle postèrieure, la seconde. Toutes les deux devait poursuivre les articulation syntactiques de l'oeuvre de l'Empereur. Celle-ci, hèlas, ne nous est pas connue que d'une manière tout-à-fait insatisffaisante; on sait quand même qu'elle comportait huit livres, le plus probable quatre pour la première guerre et quatre pour la seconde.

En observant avec soin le décor et l'action on peut s'appercevoir qu'il y a une certaine unité pour de longues ségments de la bande figufée comme aussi des changements pronnoncés tant du thèatre d'action, que des participants et du genre de cette action. Ces changements sont aussi marqués formellement par l'interruption sans équivoque de la continuitée de la bande figurée. De cette manière on peut distinguer quatre grandes sousdivisions - que nous dénommons actes - à l'intérieur de la première guerre et autres quatre dans la seconde. Le premier (1.1.) commence dans l'axe de la ordonné SE et de l'horizontale du tore de la base de la Colonne et est clos par le Danube traversé par les daces et leurs alliés sarmates qui passent en Moèsie; le second (1.2.) commence au Danube traversé par les daces et finit au Danube sur lequel l'armée romaine embarquée navigue vers la Dacie; en fin le troisième (1.3.) commence après la navigation mentionnée auparavant par la

\footnotetext{
11 v.plus haut $n .2$

12 v.plus haut n.1

13 v.plus haut $n .5$

${ }^{14}$ Gauer Werner. Untersuchungen zur Trajanssäule. vol. I, Berlin, 1977
} 
traversèe du Danube de par l'armèe romaine sur un pont de bateaux et son fin est marquè par l'apparition des forteresses dades; le quatrième acte commence avec le premier siège d'une citadelle dace et finit avec la Victoire écrivant sur un bouclier (1.4.); la deuxième guerre (2) commence avec un acte (2.1) representant la navigation de l'armée romaine depuis l'italie jusqu'à un port maritime au voisinage de la Dacie; Le second acte se deroule dans un paysage montagneux et boisé aux allentours des forteresses royales daciques et des garnisons romaines $y$ siégeant (2.2.); le troisième commence au Danube que les romains passent par le pont de Drobeta et finit avec la conquêtte de Sarmizegetusa Regia (2.3.); en fin le quatrième commence à Sarmizegetusa et finit avec les troupes romaines qui éscortent les paysans daces qui retournent aux leurs foyers abbandonés pendant les combats (2.4.). Chaque acte est suscéptible d'être divisé eh plusieurs épisodes qui repésente une sous-action déterminée, comportant à son tour une sousdivision en des séquences qui correspondent dans la plupart des cases aux scénes de Cichorius et que nous-même désignerons du ce nom (Tableaux 5,6). L'illustre savant allemand n'a pas quand même saisi les compositions compléxes de type panoramique ou travelling que Allain Mallissard a su si bien mettre en valeur et ça a bien influencé sa partition. Dans la discussion qui suit nous allons tenir compte des observations pertinentes de Malrissard, ce que modifiera aussi la partition des scènes que la hièrarchisation sintactique et sémantique de celles-ci. Mais on laissera de coté les identifications topographiques qui font l'objet d'un autre étude, comme aussi on ne prêtera guerre attention aux marques "calendaristiques" - comme par exernple la parutions des certaines végétales qui ont des tèrmes detèrminés de maturation auxquelles an accordera la due attention ailleurs et autre fois.

2.2.1. Le premier acte de la première guerre dacique commence du zéro physique de la bande à figures et son fin est formellement marqué par un arbre qui barre toute la bande figurée. L'arbre barrant toute l'hauteur de la bande figurée, avec le sens de marque de la fin d'une action, a déjà été remarquée par Cichorius ${ }^{15}$. Seulement, à la fin du premier acte il y a deux arbres qui barrent toute l'hauteur de la bande figurée, encadrant une scène a caractère spécial ( $X X I X / X X X=1.1 .4 .4$.). Cette scène est clairement limitée à gauche et à droite par un arbre/barre et divisée a mi-hauteur par une crête montagneuse plus ou moins horrizontale. Dans la moitié inferieure des soldats romains combattent quelques daces qui - paraît-il - éscortent trois daces inérmes, le premier un homme jeune et sans barbe, l'autre plus agé et barbu et un garçon. Au dessous de ce groupe dens une alvéole peut-étre une cave - du bétail mort. Ces trois se dirigent vers une passe qui paraît traverser les montagnes, assurant la liaison avec la moitié superieure. Celle-ci est occuppée au centre par un group de femmes portant des enfants, tant garçon que filles qui se dirigent vers une barque ammarée a la berge d'une rivière (?). En tête de ce groupe est une femme dace rélativement isolée - ce qui veut signifier sa position préeminente parmi les autres. À gauche, de l'autre coté de la crête montagneuse, un group de trois chevaliers romains dont le premier porte une torche - signe d'une cavalcade hattée, de nuit ${ }^{16}$. Entre les chevaliers et les femmes daces, orienté sans équivoque vers ces dernières,

\footnotetext{
${ }^{15}$ Cichorius. Op.cit, p. 141, Erklärung, Bild XXIX; cf. Lepper, Frere. p.76.

${ }^{16}$ Lintérprétation, tant de Cichorius. I, p.141, que de Lepper \& Frere, p. 76-77 qui tous les trois voient dans les trois chevaliers des soldats romains incendiateurs des établissements daces ne se confirme pas par la comparaison avec les autres scènes ( LVI, UX, CXIX, CLII) dans lesquelles apparaissent des incendiateurs. Dans toutes cettes scenes les "incendiateurs" sont à pied et complétement armés - dans la scène $X X I X / X O X$ il leur manque la casque. En échange, le premier porte un objet à franges sur le flanc droit - peut-être une poche (à lettres). Les "incendiateurs" ont dans toutes les autres quatres scènes mentionnées un mouvement normal -pas hatté - et une attitude sans équivoque par rappport de l'objet à incendier. Dans la scène XXIX/XXX tout ce qu'on peut dire ce que les trois chevaliers sont en mouvement rapide - galop - paralleiment à deux amples édifices raccourcis à cause de l'éspace disponible. En fin, dans toutes les quatres autres scènes on peut voir les flammes qui jaillissent des bátisses incendiés. Il n'y a pas de trace de flamme dans la scène $X \times 1 \times / X \propto X X$. En échange l'identification des trois chevaliers avec des veredarii (Pannonii ou non) proposé par Cichorius, I, p. 141 corrobore l'hypothèse du méssager, étant donné que les
} 
l'empereur, accompagné par un autre haut officier, qui tend la main ouverte vers la première des femmes daces.

La scène n'a aucune continuité d'action ni avec la précédente qui - ayant un caractère spécial qu'on va discuter autre part - représente deux récéptions des ambassades daces, ni avec la scène succéssive qui raconte le passage du Danube à la nage par les daces et leurs alliés sarmates. Il est plausible de rapporter le groupe des daces inèrmes, mâles et femmes, au bien connu passage de Diu Cassius ${ }^{17}$ qui mentionne la capture de la soeur de Decebale par Laberius Maximus. Il semble donc que le Maestro a réuni quatre séquences simultanées et reliées dans une et seule scène.Son contenu pourra être ainsi dêcrit: tandis que Trajan êscorte au navire qui doit la transporter à Rome la soeur de Decebale récemment capturé par Laberius Maximus, un méssager arrive l'annonçant que les daces et les sarmates ont envahi la Moesie Inferieure.

Il s'agirait donc d'une éspece de scène de transition légerement reliée à la précédante - la capture des prisonniers est le résultat d'une action sécondaire de la campagne presentée dans les premières XXVII scènes - et qui éxplique ce qui s'ensuit - la campagne moesique des romains. Son tèrme final est marqué par le second arbre-barre de la scène $X X X=1.1 .4 .4$.

Le premier épisode (1.1.1.) commence au point zèro physique de la bande dessinée et il est clos par le Danube, ainsi comme il est figuré dans la scène du passage du fleuve par l'armée romaine (IV = 1.1.1.4.). Il en couvre quatre scènes: la première $(I=1.1 .1 .1$. ) reprèsente deux bourgs (burgi) faisant partie du Limes dannubianus de la Moesia - le plus probable Inferionis ${ }^{18}$. La seconde (I = 1.1.1.2.) est assez ressemblante à la première; seulement elle représente trois burgi comme aussi les troupes les garrnisonant. Un des trois burgi - le premier dans le sens du déroulement de la naration sculptée - est flanqué vers la gauche d'une pile de bois - qui d'ailleurs marque le commencement de la scène (NE1 $\Leftrightarrow 0)$ - et de deux meules de foin. Tous les trois burgi sont flanqués par de soldats auxilliaires et sont prevus d'un balcon périmétral sur consoles en bois, à l'entrée frontale duquel est fixée une torche: un signal lumineux. Comme la scène suivante représente le déchargement des provisions et furnitures militaires des bateaux arrivés sur le fleuve, il est possible que les trois torches repprésentent le balisage de la voie navigable. Le dernier auxilliaire de droite marque la fin de la scène (NV1 $\Leftrightarrow 0$ )

La troisième scène ( $\mathrm{II}=1.1 .1 .3$.) - celle du déchargernent des bateaux - assez complèxe comme décor - deux ligne de sol, quatre édifices groupés en deux enceintes closes par des palissades circulaires (?) - finit au rupture du décor Inarqué par l'appariton d'une roche, bordée par un arbre solitaire - qui, d'ailleurs, fait fonction de signe de division - et finissant en plateau sur lequei est représentée une ville. Ainsi donc, la fin de la troisième scène est indiquée par l'arbre susmentioné (SV1 $110 \mathrm{mb}$ ).

La quatrième scène (III-IV = 1.1.1.4.) représente le passage du Danube par l'armée romaine, inclusiv la présentation de la ville port sur Danube constituant le point de départ du corp éxpéditionnaire ${ }^{19}$, commence à l'arbre susmentionné et est close par la fin du pontin qui relie le pont

\footnotetext{
troupes des veredarii etaient nottament chargé de la poste militaire - cf. Tudor Dumitru. Encidopedia civilizabief romane. Bucureşt, Editura știinţifier şi Enciclopedică, 1982, s.v.

${ }^{17}$ Dio Cassius. LXVIII,9.4.

19 Les localisations des differantes sites représentés sur la Colonne faít l'objet des controvèrses assez animées et pour celte raison seront discutées dans une autre ètude.

19 L'image de la ville ne peut être constitué en une scène autonome: il est vrai qu'elle présente une certaine cohèrence compositionelle mais sans la continuation de la séquence du passage du Danube elle se présente comme "une place ou rien ne se passe".
} 
proprement dit à la terre ferme. Fait remarquable: les deux officiers supérieurs formant la tête de la colonne, comme aussi le tubicen corréspondant comme position du second régistre, tournent leurs chefs vers arrière, mouvement en opposition avec celui des chevaliers à pied qui les devancent ${ }^{20}$, indiquant ainsi la fin de la scèrie (SE1 $\Leftrightarrow 0$ ).

Le second épisodé (1.1.2.) finit aussi par un "cut": lés soldats harangués par l'empereus Trajan ( $X=1.1 .2 .4$.) tournent carrément leur dos auX constructeurs des fortifications de la scène suivante (XI = 1.1.3.1.). Il comprend quatre scénes constituant l'inauguration de la campagne: conseil de guerre (1.1.2.1.); lustration (1.1.2.2.); présage (1.1.2.3.); harangue (1.1.2.4.). La première scène (1.1.2.1.)commence de la fin du pontin mentionné anterieurement et finit à l'arbre qui suit le dernier chevalier (et le dernier cheval) de droite (NE2 $\Leftrightarrow 0$ ). Déterminée de cette manière 'elle comprend les scènes V, VI et VII de Cichorius. Il y a un indice important de la continuitée d'éspace et d'action des trois séquences delimitées par le savant allemand: la troupe de chevaliers portant des lances passe par derrière le suggestus sur lequel est assemblé le concilium. Donc on pourra traduire en terms litteraires l'image - faite, il est vrai des trois séquences distinctes - comme: pendant que les chevaliers de telle Alla avancent en terre ennemie, lempereur tient conseil de guerre.

La seconde scène (VII = 1.1.2.2.) est une /ustratio. Elle commence juste après l'arbre susmentionné et finit à un autre arbre au delà duquel apparaît un chevalier "barbare" desarçonné (NV2 $\Leftrightarrow 0$ ). Celui-ci notamment et le groupe sur le suggestus - c'est à dire l'empereur acompagné par deux officiers généraux - forment ensamble la troisième scène (IX = 1.1.2.3.) dont la fin est marqué par l'opposition de sens des deux groupes impériaux (NV2 $\Rightarrow$ ). Nous ne nous attarderons point sur le sens de cette scène, nous nous contentant de déclarer que nous la considerons - bien dans la tradition des historiens romains - comme l'image d'un prèsage, plûtot que celle d'un messager portant une lettre écrite sur un champignon ${ }^{21}$.

La quatrième scène ( $X=1.1 .2 .4$.) est l'image d'une harangue et son fin est clairement indiqué par l'apparition d'un autre élément de paysage: le long mur qui est en train d'être construit par les legionnaires (SV2 $\Leftarrow 7 m b 2^{*}$ ). Elle est composée de deux groupes antithétiques: le groupe de l'empereur et des deux officier monté sur le suggestus et le groupe compact des soldats attentifs au discours de leur chef suprême.

Le troisième épisode (1.1.3.) est constitué de quatre scéries aussi, est son fin est indiqué par l'apparition d'une forêt qui est deffriché par les légionnaires juste an l'axe SE du tambour II'(spire 3). Dans l'épisode suivant (1.1.4.) il s'agît d'un grand combat et de ses conséquences, donc d'un changement radical de l'action.

La première scène $(X I, X I I, X I I I=1.1 .3 .1)$ a comme facteur commun une longue muraille continue, interrompue de trois (?) portes et coupant au moins un cours d'eau. À l'intérieur de cette muraille on peut distinguer deux camps romains. La longue muraille dêcrit un coude dans l'éspace libre duquel apparaît l'empereur accompagné des deux officiers généraux. À l'éxterieur de ce coude est situé un autre camp gardé par des prétoriens. La fin de la scène est marqué par le soldat maniant un pic (SE2 $\Leftrightarrow 0)$.

La seconde scène (XIV, XV = 1.1.3.2.) a comme facteur commun un paysage plus mouvementé que le précédent, plûtot plane: le tracée de l'armée passe au moins trois ponts sur des

\footnotetext{
${ }^{20}$ Líndication de la division des deux scénes par deux images similaires mais opposées comme diréction du mouvement propre, a été identifié par Alain Malissard. (1972.579) avec le "cut" cinématographique.

${ }^{21}$ Cf. Cichorius. op. cit Bild IX, p.52 ; et Vulpe Radu. Columna lui Traian. Bucuresti,
} 
cours d'eau, parcourt une route en serpentines et traverse un forêt par lequel les legionnaires doivent couper le chemin. Sa fin est notamment la fin de la forêt, après laquelle le paysage change de nouveau (NE $3 \Leftrightarrow 0)$.

La troisième scène (XVI, XVII, XVIII, XIX = 1.1.3.3.) a comme facteur commun un paysage montagneux, avec l'apparition des connifères et une ligne du terrain montante. Elle est composée de quatre séquences, dont deux centrées sur la figure de l'empereur. Toutes les quatres sont de séquences de constructions des camps par les legionnaires. La première séquence impériale represente l'empereur parlant au soldats-constructeurs; dans la seconde séquence impériale un prisonnier dace est presenté à l'empereur. La fin de la scène est marqué par une paire de connifères $\left(\mathrm{NV} 3 \Rightarrow 4 \mathrm{mb} 2^{\prime n}\right.$ ). C'est une disposition qui peut être identifiée le plus vraisemblable au travelling lateral d'Alain Malissard 22 .

La quatrième scène $(X X, X X I, X X I I=1.1 .3 .4$. $)$ a comme facteur commun toujour un paysage motagneux mais la ligne de terrain est descendante. La route commence partant d'un camp situé au changement de pente. À l'intérieur du camp apparait l'empereur qui scrute le thèatre de l'action. On peut considerer que cette séquence fait fonction commune pour les deux scènes voisines (1.1.3.3. şi 1.1.3.4.). Suivent d'autres sèquences: la cavalerie se met en marche (a), la marche de la cavalerie par devant une fortification (b), la marche de l'infanterie lourde (les légions) passant auprès d'une forteresse dacique $(c)$ et son arrêt à la lisière d'un forêt $(d)(S E 3 \Leftrightarrow 0)$.

Le quatrième épisode du premier acte (1.1.4.) nous raconte en images la première grande bataille contre les daces et la poursuite. Le paysage reste un paysage mouvementé et boisé. Il y a à signaler deux détails topographiques: une forteresse dace située sur une hauteur et une large riviére coudée. La végétation consiste en général en des arbres feuillus, mais au commencement de la scène comme aussi à sa fin on peut discerner aussi des connifères.

La première scène (XXIII, XXIV = 1.1.4.1.) commence avec le passage de l'armée romaine par le forêt (a), poursuit avec la concentration des troupes près d'une forteresse, plus probablement dacique et éventuellement abbandonée (b), puis avec l'assaut de la cavalerie (c) et est ctose par la grande melée à la quelle, du coté des romains, participe Jupiter Fulminans lui-même (d) et finit par la retraite de daces dans un forêt de conifères $(e)(N V 4 \Leftrightarrow 0)$.

La seconde scène $(X X V, X X V I=1.1 .4 .2$.) raconte les événements suivant immédiatement la bataille: l'empereur qui inspécte une forteresse dacique abbandonnée (a), les daces qui se retirent de la forteresse pour se regrouper ailleurs (b), et l'armée romaine qui traverse une grande rivière par un gué amplacé dans une coudée du cours d'eau, près d'une contrée rocherıse (c). La limite de la scène est indiquée par le sens contraire du mouvement des acteurs - soldats passant le gué et soldats participant à la harrangue de l'empereur qui se tournent carrément les dos (SV4 $\Rightarrow 0$ ).

La troisième scène (XXVII, XXVIII = 1.1.4.3.) doit être situé aux étapes suivantes car on peut distinguer deux camps d'étape comme éléments de décor. À la première, l'empereur, accompagné par deux officiers généraux et monté sur un suggestus harrangue les troupes assemblées à l'intérieur du camp (a); pendant le discours arrive, conduite par deux gentiles gérmaniques, une ambassade de comati daces (b). Devant un camp (un autre camp que celui antérieur ou le même ?) l'empereur mene des pourparler avec une ambassade de comati daces (c). La fin de la scène est indiquée par un arbre-barre: un connifère (SE4£ $4 \mathrm{mb})$.

\footnotetext{
$z_{\text {Idem. Op.cit p. } 547}$
} 
La quatrième scène $(X X I X, X X X=1.1 .4 .4$.) a déjà été analysée au commencement de ce chapitre et il n'y a aucune necessitée de reprendre ici la discussion. Retenons que un arbre-barre toujours un connifère - marque la fin de la scène, de l'épisode et aussi de l'acte (NE5ץ16 1*).

2.2.2. Le commencement de l'acte suivant (1.2.), comme aussi sa fin, est indiqué par la figuration du fleuve Danube sur lequel l'armée romaine se deplace en navires. La plûpart des éxégètes de la Colonne ont admis que cet acte constitue la narration figuré des luttes qui ont eu lieu en Moesie Inférieure à la suite d'un contrattaque des daces alliés aux sarmates sur le territoire de la province romaine. Tenons le pour acquis et poursuivons l'analyse du découpage de la narration figurée. Le second acte comporte seulement trois épisodes.

Le premier épisode (1.2.1.) peut être caracterisé comme celui qui narre la concentration des combattants sur le thèatre de gueme et contlent trois scènes. Il commence juste après le second arbre-barre susmentionné et finit avant la séquence du débarquement à la ligne formée par l'opposition des orientations des figures des soldats romains - rameurs des navires et déchargeurs des bateaux - qui, comrne d'habitude se tournent les dos.

La première scène $(X X X I, X X I I=1.2 .1 .1$.) représente le passage du fleuve à la nage par les daces et par leurs alliés, les chevallers sarmates. Il n'est pas ici le lieu de discuter sur la thème de la modalité du passage - à la nage, sur la glace. Retenons que tant les chevaux que les personnages humains apparaîssent mouillés dans les eaux du fleuve. On peut aussi distinguer trois séquences distinctes: la concentration des daces et sarmates sur la rive gauche auprès d'une fortification probablement dace (a), le passage et la lutte avec les vagues (b) et en fin l'abordage à l'autre rive et l'assaut d'un camp romain par les daces fraichement passés, dans une composition concentrique, très claire. La fin de la scène est marqué par un arbre-barre (NV5 $=3 m b 2^{\prime \prime}$ ). Il s'agit, probablement de la neutralisation du camp gardant le gué par lequel les daces et les sarmates sont passés sur l'autre rive.

La seconde scène (XXXIII =1.2.1.2.) a cornme lieu de nouveau la rive gauche: il est representé comme une ville-port dotée d'un quai d'embarquement et de deux arcades, dont une est située apparêment sur la rive droite. La thème de notre étude ne nous permet pas de nous attarder sur les détails topographiques, d'ailleurs très importants et non moins significatifs dans le cas de la scène en discussion. Sur le quai l'armée romaine, l'empereur en tête, s'embarque en des navires de guerre et de transport. La limite de la scène est marquée par les arcades susmentionnées (SV5 $\left.11 \mathrm{mb} 3^{\prime n}\right)$.

La troisième scène (XXXIV, XXXV = 1.2.1.3.) raconte la navigation de l'ammée sur le Danube et le débarquement sur la rive droite. L'opération a lieu dans un port prévu d'une fortification. Le coté droit de cette fortification prolongée par la ligne de dos d'un personnage qui, se mouvant en sens opposé reçoit l'empereur, indique la fin de la scène (SV5 $\Rightarrow 20 \mathrm{mb} 1^{m}$ ). On peut aussi distinguer plusieurs séquences: la navigation des bagahges et des chevaux (a), la navigation des troupes (b), le' débarquement $(c)$. Avec cette scène finit aussi l'épisode.

Le second épisode (1.2.2.) presente les premiers combats proprement-dits de la Moesie Inférieure et son fin est indiqué par un arbre-barre le séparant de la scène de la harrangue adressée par l'enipereur à l'armée. L'épisod est constitué de quatre scènes.

La première scòne (XXXVI $=1.2 .2 .1$.) représente la marche forcée de l'armée romaine vers le champ de bataille. Son tèrme est indiqué par un arbre barre $(\mathrm{SE5} \Rightarrow$ ). La seconde scène (XOXVII = 1.2.2.2.) raconte un combat de cavalerie; elle est close aussi par un arbre-barre (NE6 $\Rightarrow 10 M B)$. 
troisième scène (XOXVIII = 1.2.2.3.) présente une melée generale autour d'une forteresse de chariots: les troupes auxilliaires annéantissent les barbares attaquants; son tèrme est aussi marqué par un arbre-barre (NV6 $\Rightarrow 9 \mathrm{mb} 3^{\text {in }}$ ).

La quatrième scène (XOXIX $=1.2 .2 .4$.) a un caractère complexe: au centre l'empereur à l'intérieur d'un camp roraain en cours de construction reçoit une délégation des nobles daces; à l'éxtérieur, à la gauche une population dace paisible, à la droite des prisonnièrs dace. Il n'y est pas la place de discuter l'éxégèse de cette scène. Qu'il nous soit permis d'observer qu'en ce qui concérne tant la délégation des nobles daces que la population dace paisible il est possible de s'agîr des représentants de la population pérégrine de la province de Moesia Inferior qui, on le sait, était d'origine dace. La ligne du coté aroit du rempart du camp romain prolongée par la démarcation indiquée par la direction opposé d'action des differents groupes des soldats romains - les gardes.des prisonniers, comme aussi ceux-ci même, tournés vers la gauche, c'est à dire vers l'empereur, les autres appartenant soit au corp d'armée en marche vers la bataille, soit aux groupes des blessés, tournés vers la droite, dans le sens de l'avance - marque la fin de la scène et de l'épisode (SV6e $\left.4 m b 2^{\prime n}\right)$.

Le troisème épisode (1.2.3.) présente la grande bataille décisive de la campagne moesique. La première scène $(X L=1.2 .3 .1$.) se developpe sur deux régistres, tous les deux aboutissant à la figure de l'empereur représenté recevant un prisonnier dace (SE6 $\Leftarrow$ ). Le mouvement en sens opposé de ce prisonnier et de sa garde crée la cèsure formelle qui sépare cette scène de la suivante. La seconde scène ( $\mathrm{XL}=1.2 .3 .2$.) est constituée par la grande bataille elle-même et son tèrme est indiqué par un arbre-barre $(\mathrm{SE} 6 \Rightarrow)$. La troisième scène (XLI = 1.2.3.3.) figure le camp de bataille après la fin des hostillités, jonché de cadavres; un petite group de daces en fuite apparait das le quart supérieur de la bande figuré. Un arbre-barre, marque la fin de la scène (SE6 $\Rightarrow 20 \mathrm{mb}$ )

Le quatrième épisode (1.2.4.) illustre les suites des batailles. La première scène (XレI = 1.2.4.1.) illustre la harrangue d'après la bataille, adressée par l'empereur à l'armée victorieuse. Son tèrme est indiqué par la ligne du coté gauche du rampart d'un camp dans lequel sont detenus des prisonniers daces $(N E 7 \Rightarrow)$. La seconde scène (XLIII $=1.2 .4 .2$.) est constituée notamment par les prisonniers daces à l'interieur du camp dont le coté gauche en forme la limite $(N E 7 \Rightarrow)$.

La troisième scène (XLIV, XLV = 1.2.4.3.) est formé des plusieurs groupes gravitant autour de l'image impériale: Trajan assis sur sella castrensis qui distribue des récompenses aux soldats. De gauche à droite se suivent les séquences illustrant la joie de la victoire: deux soldats - probablement des amis - qui s'embrassent (a), d'autres soldats qui saluent ou acclament l'empereur (b), en fin le très controversé groupe (c) des femmes daces (?) qui torturent (?) des prisonniers romains (?) $)^{23}$. Retenons que il n'y a pas de changement de décor - c'est à dire de thèatre de guerre - que les femmes pérégrines de la Moesie devaient ressembler aux femmes daces comme costume et que les. prisonniers - en tant qu'on peut observer - n'ont pas de figures romaines. La fin de la scène est indiquè par le changement de paysage - appataît le Danube - comme aussi par l'opposition des directions des actions des acteurs $(\mathrm{NV} 7 \Rightarrow)$.

La quatrième scène $(X L V I, X L V I I=1.2 .4 .4$.) est occupée par la représentation du transfert des troupes romaines du front moesique sur le front dacique et comporte deux séquences: l'embarquement (a) et le dèbarquement (b) étant un parfait éxemple de composition contractée. Son

${ }^{23}$ Cichorius. Op.cit Bild XLV, p. 217-218. Lepper \& Frere. Cp.cit. p. 90. 
Quelques observations sur le tracee et la partition de la bande a reliefs histories de la Colonne Trajane

tèrme est indiqué par la ligne du coté gauche du portail du pont des bateaux par lequel l'armée romaine traverse de nouveau le Danube (SV7 $\Leftrightarrow 0)$. C'est aussi la fin de l'épisode et de l'acte, par ce que l'apparition du fleuve et du pont signifie un changement radical du theatre de la guerre.

2.2.3. Le troisième acte (1.3.) raconte la deuxième avance de l'armée romaine à l'interieur de la Dacie aprěs san retour de la Moesie Inferieure. Il est constitué de quatre épisodes et est clos par un arbre-barre situé avant l'axe SE 10. Après cet arbre commencent les scènes d'assaut des forteresses daces.

Le premier épisode (1.3.1.) illustre le ragrouspement des troupes romaines sur la rive gauche du Danube, en Dacie, mais d'après toutes les apparences dans la partie déjà occupée par les romains assez loin de l'armée dace.

La première scène (XLVIII = 1.3.1.1.) représente la traversée du Danube par l'armée romaine sur un pont de navires. Les images d'un officier et d'un signifère qui tournent la tête vers l'arrière marquent la césure entre la scène en discussion et la suivante (SV7 $\Rightarrow 16 \mathrm{mb} 1^{*}$ ).

La seconde scène (XLXX = 1.3.1.2.) est constituèe de la figuration du marche de l'armée romaine le long des fortifications continues que nous avons vues en cours d'être construites dans la scène 1.1.3.1. La ligne verticale du coté gauche d'une fortification circulaire clôt la scène (SE7 $\Leftrightarrow 0)$.

La troisième scène (XLIX, $L=1.3 .1 .3$.) raconte l'accueil de l'armée qui a passé le Danube par l'empereur. La fin de cette scène est marquée par un traçé en ligne brisée qu'on a rencontré aussi dans la scène 1.1.3.2. et qui peut être la représentation d'un limes en vue aérienne. Le tèrme de cette scène est indiqué par la ligne des crêtes des motagnes(SE7 $\left.\Rightarrow 16 \mathrm{mb} 2^{\prime \prime \prime}\right)$.

La scène suivante, la quatrième $(U=1.3 .1 .4$.) présente la situation symmétriquement opposée de la précedente: l'empereur en tête des troupes est accueilli par un autre corp d'armée qui arrive venant du sens contraire de l'avance des romains en général. La limite de la scène est constituée par un arbre-barre (NE8 $\Leftrightarrow 0$ ), d'ailleurs le premier d'un group signifiant un forêt.

L'épisode suivant (1.3.2.) se compose aussi de quatre scènes et illustre les premiers contact avec les daces de l'armée qui avance continuellemment. La première scène ( $L I=1.3 .2 .1$.) à plusieurs séquences, présente la récéption d'une ambassade dace - encore des comati - par l'empereur (a), tandis qu'un group des legionnaires defrichent un forêt, pour ouvrir le chemin(b), d'autres legionnaires construisent une route par la montagne (c), et tout en haut on peut entrevoir une forteresse dace (d). La césure entre les deux scènes est marquée par l'oblique déterminée par le mouvement contraire des trois groupes d'acteurs: ambassadeurs daces et legionnaires construisant la route vers la gauche et soldats romain participant a une cérémonie réligieuse de la scène suivante vers la droite (NV8 1").

La seconde scène (UII = 1.3.2.2.) représente une lustratio d'avant les combats. Elle jouit d'une composition centrée et par l'effet de cette disposition les acteurs marquent, par la direction de leur action, opposée a celles des acteurs des autres scénes, la fin de la scène(NV8 $\left.\Rightarrow 24 \mathrm{mb} 1^{\prime \prime \prime}\right)$.

La troisième scène (LV = 13.3.3.) est la bien connue scène d'éxhortation à composition aussi centrale et les limites facilement reconnaîssables (SV8 $=13 \mathrm{mb} 3^{\prime \prime \prime}$ ). La quatrième (LV, LVI = 1.3.3.4.) présente la dernière étape de la marche de l'armée romaine avant de pénétrer dans le pays ennemi propnement-dit. Sa limite est marquée par la ligne du coté droit d'un camp romain, prolongée par la hampe d'une pique dans laquelle est enfoncée la tête d'un dace (SV8 $\Rightarrow 15 \mathrm{mb} 1^{*}$ ). 
Le troisième épisode (1.3.3.) est composé de quatre scènes assez variées mais toutes ayant comme trait commuu le contact direct avec les daces. Dans la première scène (LVII, LVIII, IIX = 1.3.3.1.) des soldats romains incendient des constructions daces parsemées dans un paysage motagneux, tandis que l'empereur passe sur un pont et l'armée dace observe les agissements des romains d'une certaine distance, de la motagne. La fin de la scène est marqué par un arbre-barre (NE9 $\left.\Leftarrow 16 \mathrm{mb} 3^{\prime \prime \prime}\right)$.

La seconde scéne $(L X, L X I=1.3 .3 .2$.) a plusieurs séquences. Celle centrale représente l'empereur recevant la soumission d'un chef dace défécteur (a). Les autres sont la construction d'un camp (b), le quartier général de l'empereur (c) et un corp d'armée faisant la jonction avec la force principale de sous la conduite de l'empereur (d).La ligne sinusoïde d'une crête montagneuse (NE9 $\Rightarrow$ $14 \mathrm{mb} 1$ '") fait office de césure par rapport de la scéne suivante, dont les acteurs les plus proches se dirigent en sens contraire par rapport à la troupe de la séquence (d).

La troisième scéne (LXII = 1.3.3.3.) comporte aussi plusieurs séquences, de gauche à droite: transports militaires en montagne (a), des patrouilles arrivent en vue des édifices monumentaux daciques (b), un camp romain gardé (c), des troupes auxilliaires en attente (d). Une crête rocheuse oblique descendente fait fonction de ligne de séparation entre les deux scénes voisines(NE9 $\left.\Rightarrow 16 \mathrm{mb} 3^{\prime \prime \prime}\right)$.

La quatrième scéne (LXII = 1.3.3.4.) represente la principale force d'attaque romaine concentré dans une région montagneuse, l'empereur en tête, monté sur une petite colline, accompagné des deux officiers généraux. Un arbre, comme aussi un changement du terrain (une crête rocheuse horrizontale sépare la bande figuré en deux régistre semblants) constitue le signe du découpage des deux scénes voisines et constitue à la fois l'indice de la fin de l'épisode (SV $9 \Leftrightarrow 0)$.

L' épisode suivant (1.3.4.) est est celui de premières rencontres. La première scéne (LXIV = 1.3.4.1.) représente la cèlébre charge de la cavalerie maure de Lusius Quietus. Elle finit par un arbrebarre, le dernier d'un group représentant un forêt (SE9 $\Rightarrow 3 \mathrm{mb} 1 *$ ).

La seconde scéne (LWV, LXVI stg. = 1.3.4.2.), comportant plusieurs séquences est centrée sur la figure de l'empereur qui reçoit la soumission des deux pileati daces (a); de gauche à droite: construction d'un camp (b), transport des machines de guerre (c), le quartier général de l'empereur (d). La ligne des troupes romaines defendant un rempart de bois, orientée en sens opposé par rapport au groupe des chefs daces, leur voisins, marque le tèrme de la scéne (NE10 $\Leftrightarrow 0$ ).

La troisième scène (LXVI centre et dr., LXVII = 1.3.4.3.) illustre un contrattacque des daces dirigé envers le quartier général de l'empereur. Les troupes romaines improvisent un rempart de bois sur lequel sont emplaçées les machines de guerre. Les attacants descendent une hauteur boisé couronnée d'une forteresse dàcique. Un arbre-barre fait la fonction de ligne départageant les scènes (SV10 $=5 \mathrm{mb}$ ). En fin la dernière scène de l'épisode, comme aussi de l'acte (LXVIII = 1.3.4.4.) a plusieurs séquences, dont la principale est la présentation d'un pileatus dace prisonnier devant Trajan (a); de gauche à droite: des legionnaires construisant un camp (b), une patrouille (c), legionnaires defrîcheant un forêt. La césure est représentée par un arbre-barre (SE10 $\left.=9 \mathrm{mb} 3^{\prime \prime \prime}\right)$.

2.2.4. Le quatrième acte (1.4.) constitue la narration en images des combats décisives menés dans les Carpathes et de la victoire finale des romains. Il est composé de quatre épisodes, chacun à deux scènes compléxes comportant plusieurs séquences. Le premier trophée, de gauche, de la scène de la Victoire écrivant sur un bouclier, fait la fonction de limite de l'acte comme aussi de la première guerre. 
Le premier épisode (1.4.1.) presente l'assaut d'une forteresse dacique ${ }^{24}$. La première scène (1.4.1.1.) raconte la bataille sous les murailles de la forteresse. Elle est limitée par un arbre-barre $\left(\mathrm{SE} 10 \Rightarrow 16 \mathrm{mb} 3^{\prime \prime \prime}\right)$. La seconde scène (LXXI = 1.4.1.2.) illustre l'assaut de la forteresse par les legionnaires ayant formé la testudo. Son tèrme est indiqué par une crête arquée de montagne qui sépare un group de soldats participants passifs à l'assaut de la première forteresse du groupe: de l'empereur (NV11 $\Leftrightarrow 0$ ). Le second épisode (1.4.2.) consiste de nouveau dans l'illustration de la conquête d'une autre forteresse. La première scène (LXXII = 1.4.2.1.) presente la bataille dans la présence de l'empereur (a) et plusieurs autres séquences - des soldas presentant les têtes coupés des ennemis à l'empereur (b). les legionnaires ragrouppés en vue de la bataille (c). les auxiliaires en lutte avec les daces (d). enfin, dans le régistre supérieur, l'armée dace abbandonant la forteresse (e). La fin de cette scène est indiquée par un arbre-barre (NV11 $\Rightarrow 6 \mathrm{mb}$ ). La seconde scène (LXXIII =1.4.2.2.) est la représentation d'une harrangue de l'empereur. La muraille d'enceinte d'une forteresse - plus probablement dacique - à l'intérieur de laquefle a lieu le rassemblement des troupes pour écoutes la harrangue isole la scène (NV11 $\Rightarrow$ ).

Le troisième épisode (1.4.3.) raconte des événements dont l'importance, faute d'information littéraire, éxplicite, nous échappe ou il est possible seulement être supposée. La première scène (LXXIII éxtérieur = 1.4.3.1.) presente des legionnaires qui, è l'éxtérieur de la fortification dans laquelle a lieu la harrangue, défrichent un forêt et ouvrent un chemin. La scène est delimitée par un arbre-barre (NV11 $\Rightarrow 16 \mathrm{mb} 2 " ')$. La seconde scène ( $L X X V$, LXXVI régistre supérieur = 1.4.3.2.), comportant deux séquences, décrit un détachement de cavaléristes romains dans la présencel d'un cours d'eau, petit mais abbondant, coulant dans un bassin carré (a), tandis que un group de legionnaires penétrent, transportant des bagages dans une forteresse dacique (b). Le tèrme de la scène est indiqué par le sens contraire de la diréction d'action des soldats près du cours d'eau et de ceux qui entourent l'empereur dans la scène suivante (SV11 $\Leftrightarrow 0$ ).

Le quatrième épisode (1.4.4.) - le dernier de la première guerre - a comme principale thème la soumission des daces et est suivi de la célébre scèrle de la Victoire. La première scène ( LON, LXXVI = 1.4.4.1.) représente proprement-dit la soumission et comporte plusieurs séquences: les troupes romaines faisant front (a), l'empereur siègeant sur la sella castrensis monté sur le suggestus et recevant la soumission des daces (b), un premier groupe de pileati daces, à genoux, les armes par terre implorant l'empereur (c), un second groupe habillé en daces, les mains liées au dos, mais dressés (d), un autre grand groupe des daces - comati et pileati melés - agenouillés (e), un quatrième groupe des daces dressés ( $f$ ) et en fin un grand pileatus, probablemnt le roi Décébale ( $g$ ) suivi d'un groupe de comati qui démolissent une muraille de la fortification (h), puis d'un autre groupe de pileati à l'intérieur d'une fortification (i) et la scène est close d'un grand groupe de daces civiles hommes, femmes et enfants - qui descendent d'un paysage montagneux, accompagnés des leurs troupeaux de bétail vers le groupe principal de la rédition (j). La scène est close par un arbre-barre $(N V 12 \Leftarrow 7 \mathrm{mb})$. La seconde scène (LXXVII = 1.4.4.4.) est l'image de la traditionelle harrangue de la fin de la guerre. Elle est suivie immediatemment par la scène de la Victoire sans aucune autre séparation formelle autre que l'apparition du trophée «dacique» (NV12 $=7 \mathrm{mb}$ ).

3.1.1. Après la scène de la Victoire commence la seconde guerre avec un premier acte (2.1.) qui raconte la navigation de l'empereur Trajan et de l'armée éxpéditionaire romaine dépuis

\footnotetext{
${ }^{24}$ Le problème de lídentification des forteresses daciques représentées sur la Colonne Trajane constitue objet de controvèses. Nous nous porposons d'attaquer cette question dans un autre article en nous limitant de signaler aue celle représentée dans la scéne $L X X=1.4 .1 .1$. pourrait être celle de Costeşti (double enceinte - éxterieure en palissade, intérieure en muraille de pierre)
} 
l'Italie jusqu'au thèatre de la guerre. Cet acte comporte quatre épisodes à deux scènes chacun et finit avec l'arrivée de l'empereur dans une ville de la Moesie Inférieure. Chaque épisode raconte une étape de ce voyage, la premiére scène décrivant le point de départ et la navigation, la seconde illustrant l'arrivée: topographie et action. L'identification des villes et ports constituant les étapes de la route de l'empereur a donné l'occasion à bien de controverses et nous n'insisterons plus sur ce point.

La première scène $(L O X X, L O X X=2.1 .1 .1)$ du premier épisode (2.1.1.) décrit le port du départ (NV12 $\Rightarrow 10 \mathrm{mb} 3^{\prime \prime}$ )dans lequel la majorité des éxégètes de la Colonne reconaissent la ville d'Ancona, et raconte aussi la navigation sur la Mer Adrlatique (SE12 $\Leftarrow 12 \mathrm{mb})$. La seconde (LXXXI = 2.1.1.2.) décrit le point d'arrivée - une ville fortifiée, dotée d'un phare comme aussi d'autres installations portuaires, avec un plan orthogonale, des rues portiquées et des monaments importants, située paraît-il sur un promontoire. L'empereur est acueilli festivement par les citoyens mais l'action est marquée par la vitesse. La tour du phare indique la fin de la scène (SE12 $\left.\Rightarrow 12 \mathrm{mb} 2^{\prime \prime \prime}\right)$.

La première scène $(L X X X I I=2.1 .2 .1$.) du second épisode (2.1.2.) a une forme contractée : la représentation des deux navires clans l'arc d'un golfe (la ligne de la cote constitue la limite de la scène - NE13 $\leftarrow 5 \mathrm{mb}$ ) et des soldats qui s'affairent sur le pont des bâtiments tient place de la déscription détaillée de l'embarquement, la navigation et le débarquement. La seconde scène (XXXIII, XOXIV, $\mathrm{XONV}=2.1 .2 .2$.) représente l'accueil soliennel de l'empereur dans une ville côtière symbolisée, aussi par contraction, par sa porte - voisine d'une garnison romaine, avec un sacrifice sur deux autels et en présence des citoyens pérègrins de la ville comme aussi des citoyens romains couronnés et des soldats. La fin de la scène est indiquée par la ligne du coté de la muraille du camp romain voisin de la ville (NV13 $\Rightarrow 7 \mathrm{mb}$ ).

Le troisièrne épisode (2.1.3.) commence par la représentation des deux navires qui forment la première scène (LOONVI gauche = 2.1.3.1.) avec la mêrne rédaction contractée comme auparavant $(S V 13 \Leftarrow 17 \mathrm{mb} 1 ")$, la seconde scène (LXOVI = 2.1.3.2.) presentant l'accueil de l'empereur dans une ville-port fortifiée, située sur un promontoire et caracterisée par la présence d'un grand temple voisin d'un thèatre, comme aussi d'autres monuments (un petit temple, une tour, une grande bâtisse publique). Sur la place l'empereur et sa suite sont acqueilli avec un sacrifice par des citoyens romains mais aussi par des soldats en habit de voyage (une jonction des troupes?). La fin de la scène est indiquée par un pilier vertical; probablement le coté de la porte du port (SV13 $\left.\Rightarrow 1 \mathrm{mb} 3^{\prime \prime \prime}\right)$

Le quatrième épisode (2.1.4.) commence de nouveau par la représentation des navires accostées dans un port près d'un cap à pic et de l'armée, l'empereur en tête, débarquée et accueillie par d'autres troupes près d'un camp permanent - castra stativa. Cest la première scène (LOXVII, LOOVIII = 2.1.4.1.) differante comme contenu et par conséquence autrement detaillée. La fin de la scène est indiqué par la ligne du coté du rempart du carap romain (SV13 $\Rightarrow 23 \mathrm{mb} 3^{\prime \prime \prime}$ ). La seconde scène ( $L O O X I X, X C, X C I=2.4 .4$.2.) illustre le chemin depuis la mer jusqu' à une ville à l'intérieur de la province, peutêtre riveraine du Danube, peuplée tant des citoyens romains que des pérègrins, dedans laquelle l'empereur est sollennellement acqueilli par un sacrifice sur six autels. Le tèrme de la scène est indiqué par un rangée de boucliers héxagonaux qui marque aussi la fin de l'épisode et de l'acte (NV14־9mb 2"').

3.1.2. Le second acte de la deuxième guerre (2.2.) se deroule sur un tout autre thèatre de guerre - le territoire de la Dacie et est constitué des quatre épisodes variés racontant les préliminaires des grands combats ayant quand même lieu dans la zone de la Dacie controlée par les romains.La fin 
de l'acte est indiquée par un arbre-barre après lequel apparaissent des fortifications occupées par les daces.

Le premier épisode (2.2.1.) a lieu dans un paysage montagneux et boisé. L'action consiste en une sèrie des combats - assauts, batailles rangées, éscarmouches - dont l'initiative appartient aux daces et se deroulent dans des objectifs occupés par les romains. Le tèrme de l'épisode est indiqué par un arbre-barre après lequel le paysage change: les environs du pont sur le Danube de Drobeta.

La première scène $(\mathrm{XCII}=2.2 .1 .1$ ) du premier épisode située dans un environment montagneux et boisé présente des soldats mmains construisant une route en serpentine parsemée des camps et des ponts. Sa fin est marquée par un arbre-barre après lequel les acteurs changent - les daces font leur apparition (NV14 $\Rightarrow 18 \mathrm{mb} 1^{*}$ ). La seconde scène (XCIII $=2.2 .1 .2$.) représente le rassemblement des daces qui occuppent de nouveau une des leurs forteresses désafféctées ${ }^{25}$. La césure entre cette scène et la suivante est marquée par la ligne départageant deux groupes des daces avec des diréctions d'actiop opposées (SV14 $\Rightarrow 13 \mathrm{mb} 2^{\prime \prime \prime}$ ). La troisième scèné (XCIV, XCV = 2.2.1.3.) raconte les attaques daces contre les positions romaines et a deux séquences : l'attaque d'un camp romain par les daces et l'assaut des position romaines consistant en des barrages appuiés sur des formes de reliefs par les daces. La fin de la scène est indiqué par la ligne du dernier barrage (SE $14 \Rightarrow)$. En fin la quatrième scène (XCVI, XCVII = 2.2.1.4.) illustre l'intervention de l'empereur en tête d'un corp éxpéditionaire qui met fin à l'aggression des daces. Le tèrme de cette scène est indiquée par un arbre-barre (NE15 $\Rightarrow 13 \mathrm{mb}$ ), le dernier d'un group conniottant un forêt en cours de défrichage. Cest aussi l'indication de la fin de l'épisode.

Le second épisode (2.2.2.) racontant les préliminaires de la campagne, commence avec une scène (XCVIII, XCIX = 2.2.2.1.) représentant l'inauguration du pont sur le Dannube de Drobeta. Le tèrme de la scène est indiqué par la ligne du coté gauche d'un camp romain sur lequel le pont débouche (NV15 $\Rightarrow 8 \mathrm{mb} 3^{\prime \prime \prime}$ ). La seconde scène $(C=2.2 .2 .2$.) représente la jonction des troupes des gentiles symmachiarif ${ }^{26}$ (a). Cette scène est limitée par l'apparition du portail du pont de la rive gauche, sous la forme d'une barre massive (SV15 $\left.\Rightarrow 2 \mathrm{mb} 3^{\prime \prime \prime}\right)$ prolongée peut-être par un arc supportant des trophées et s'appuiant, de l'autre coté sur une seconde pille-barre ${ }^{27}$ à la fin d'une sèrie d'édifices urbaios -temples, amphithèatre - (b). C'est une scène complèxe, s'inscrivant dans le type panoramique d'Allain Malissard. La troisième scène $(\mathrm{CI}=2.2 .2 .3$.) présente la traversée du Danube par les troupes venues avec l'empereur. Sa fin devait être marquée par la pille gauche du portail du pont $(\mathrm{SV} 15 \Rightarrow)$. La quatrième scène $(\mathrm{CII}=2.2 .2 .4$.) représente la jonction des troupes venues avec Trajan, après la traversée du Dannube, avec celles qui sont restées en Dacie à la fin de la première guerre. Elle a plusieurs séquences - les troupes en marche, l'empereur en tête (a); passant par dévant un centre important (b); les troupes de la Dacie acqueillent l'empereur avec un sacrifice (c); dévant un camp (d). La fin de la scène est indiquée par un arbre-barre (NE16 $\leftarrow 14 \mathrm{mb}$ ). En général le second épisode du second acte doit être localisé aux environs de Drobeta. Le troisième épisode est situé toujours dans la zone voisine du lieu du passaçe du Dannube. La première scène (CIII =

\footnotetext{
${ }^{25}$ L'identification des forteresses daces est un sujet controversé sur lequel nous ne nous attarderons pas ici, en nous resérvant l'opinion pour une autre occasion;

${ }^{26}$ Lintérpretation selon laquelle les pérégrins armés de la scène sont des ambassadeurs des peuplades voisines de la Dacie (Lepper \& Frere. Op.cit. p.151; Cichorius. 142 et suiv. -grecs; bastames et iazyges; P.B.Porescu. Trojanssäule. p. 114115.) n'a aucun fondement ni figuratif, ni litterraire; en échange on retrouve les types - costumes, barbes et chevelures aux gentiles figurés dans les scénes suivaniles.

"Sur le relief endommagé de la Colonne on peut observer une vague tracée arquée comme aussi les faibles traces d'un autre pied-droit.
} 
2.2.3.1.) représente l'habituelle suovetaurilia de tout debout de campagne. Elle est limitée par un arbre-barre qui prolonge là verticale du coté gauche du suggestus de la scène suivante (NE16 $\Rightarrow 7 \mathrm{mb}$ $\left.3^{\prime \prime \prime}\right)$. Celle-ci ( $\mathrm{CIV}=2.2 .3 .2$.) représente une harrangue - aussi habituelle que le sacrifice au debout d'une campagne et est limitée par la ligne droite du coté gauche d'un camp en terre (NV16 $\Leftrightarrow 0$ ). Dévant celui-ci (scéne $C V=2.2 .3 .3$.), à l'intérieur d'un autre camp - cette fois en pierre - un conseil de guerre présidé par l'empereur. Le coté droit, vertical, de ce dernier camp indique la fin de la scène $\left.\mathrm{NV} 16 \Rightarrow 18 \mathrm{mb} 1^{\prime \prime}\right)$. En fin, la quatrième scène (CVI = 2.2.3.4.) raconte le départ des troupes qui groupées en deux colonnes engagent l'avance en territoire dace sur deux tracées separés par une crête montagneuse.La fin de la scène est indiquée sur la colonne supérieure par un officier et dans celle inférieure par un signifer qui, tous les deux tournent la tête en arrière (SV16 $\Rightarrow$ ).

Le quatrième épisode raconte la marche d'approche de l'amnée romaine jusqu'aux environ du noyaux de la resistence du roi Décébale. Sa fin est marquée par un group de trois arbres-barre et une crête rocheuse à-peu-près verticale et qui coupe toute l'hauteur de la bande sculptée. La première scène ( $C$ II $=2.2 .4 .1$.) représente l'arrivée de l'armée à la première étape: sur chaquriè des deux routes un camp. Les cotés droites des camps marquent la limite de la scène (SE16 $\Leftrightarrow 0$ ). La seconde scène (CVIII = 2.2.4.2.) est de nouveau une image de marche. Sa fin est indiquée par la figuration d'un seul camp, nottament par la ligne de son coté gauche (NE17€ 12mb 2"'). Il parraît que les deux colonnes de marche finissent par se joindre et le camp signale le point de jonction. La troisième scène ( $C X X=2.2 .4 .3$.) illustre l'occupation de ce camp qui devait faire, peut-être, la fonction de base d'attaaue. L'oblique du coté droit du camp marque la limite de la scène (NE17 $\Leftrightarrow 0$ ). la quatrième scène $(C X=2.2 .4 .4$.) a un caractère complèxe et plusieurs séquences, figurant les préparatifs de combats: un grand camp (a); des legionnaires moissonant (b); des legionnaires capturant des chevaux (c); des auxilliaires épiant l'ennemi (d). Cé derniers sont représentés se cachant parmi les arbres signalant la fin de la scène, de l'épisode et de l'acte (NE17 $\Rightarrow 17 \mathrm{mb}$ 3"').

3.1.3. Le troisième acte (2.3.) est celui des grandes batailles terminées par la victoire des romains. Le thèatre de guerre, montagneux et boisé est celui des forteresses daces des Carpathes méridionaux très faiblement connues au commencement du siècle, quand CICHORIUS rédigeait son livre mais relévées en détail maintenant, grâce aux fouilles entreprises sur place par l'école de Cluj. L'acte comporte quatre épisodes et finit par l'abbandon de leur principale forteresse par les daces, suivi de son occupation par les romains. Un arbre-barre en marque la fin.

Le premier épisode (2.3.1.) présente la pénétration des romains dans la zone de la capitale des daces qui, nous le savons au jour d'hui, était le centre d'un système de fortifications adapté à l'envirronement montagneux constituant une defense naturelle. La première scène $(C X I=2.3 .1 .1$.) présente une importante fortification dace, d'une structure assez complèxe, ses occupants comme aussi les combactant des avantpostes en attente de l'assaut. Le tèrme de cete scène est indiqué par un réhaussement du sol rocheuJx formant une crête (NV17 $\Rightarrow 10 \mathrm{mb} 2^{*}$ )qui sèpare la fortification d'un combat en lieu ouvert, formant la thème de la seconde scène (OXII = 2.3.1.2.). La fin de celle-ci est indiqué par un group de deux arbres-barre gémminés (SV17 $=7 \mathrm{mb}$ ). La troisième scène (CXIII gauche $=2.3 .1 .3$.) consiste dans la figuration d'un grand camp romain en terre, probablement servant comme quartier général de l'empereur et ayant une forme inhabituelle. Il s'agît parrait-il de signifier l'implantation des garnisons romaines dans le milieu du noyaux défensiv dace. Le tèrme de la scène est indiqué par les diréctions opposées des mouvements des acteurs, tous des soldats romains : les sentinelles et les portsenseigaes près du camp sont orientés vers la gauche tandis que les 
Quelques observations sur le tracee et la partition de la bande a reliefs histories de la Colonne Trajane combattants toujours romains de la scène suivante regardent et avançent vers la droite (SV17 $\Rightarrow 5 \mathrm{mb}$ 1*).

La quatrième scène ( $O$ XIII milieu $=2.3 .1 .4$.) présente l'assaut de la première forteresse dacique, plûtot fortifiée naturellement. La limite de cette scène est celle oblique de droite de la forteresse assaillie (SE17 = $8 \mathrm{mb} \mathbf{1}^{\prime \prime}$ ). En fonction de cette ligne la diréction de l'action des soldats romains change. Au delà de cette ligne commence un ensemble de séquences toutes reliées à un rempart fait des gros moellons et dont un armature en bois il lui règle les assises. Ce rempart est en étroits rapports avec une citadelle construite des blocs de pierre et prevue d'une entrée à encadrement profilé. Sur le fond de ce rempart (la citadelle y comprise) se deroulent plusieurs actions diverses ét unitaires chacune, séparées par des pauses conformément au procédé du cinématographique fondu enchaîné ${ }^{2 \theta}$. Cest tout-à-fait tentant de considerer tout cet ensernble comme un seul épisode (2.3.2.) et les diverses actions comme des scènes unitaires, mais non pas séparées.

La première (CXIII droite $=2.3 .2 .1$.) raconte en images le premier assaut, parait-il sans suite. Une pause d'action la sépare de la suivante $(\operatorname{SE17} \Leftrightarrow 0)$. La seconde scène (CXIV = 2.3.2.2.) nous présente le siège : tandis qu'on bat la muraille avec des belièrs l'empereur et sa suite obsèrvent les travaux de siège d'un camp rapproché. Cette fois le changement de la diréction du mouvement des attaccant indique la cèsure entre les scènes $(N E 18 \Leftrightarrow 0)$. La troisième scène (CXV = 2.3.2.3.) c'est celle de l'assaut général et de nouveau le changement du mouvement, cette fois-ci celui des assiégés, marque la séparation (NV18 $\Leftrightarrow 0$ ). La quatrième scène (OVI = 2.3.2.4.) présente la démolition de la forteresse par les vainqueurs. Sa fin est indiquée par un grand arbre-barre qui marque aussi le commencement d'un forêt, appartenant déjà au épisode suivant (NV18 $\Rightarrow 15 \mathrm{mb}$ ).

Le troisièmı épisode (2.3.3.) illustre l'assaut et la capture de la capitale des daces Sarmizegetusa. Il commence par une scène (CXVII = 2.3.3.1.) de préparatifs: défrichage, construction d'un rempart en bois. La ligne du coté droit du rempart marque la séparation des scènes (SV18 $\Rightarrow$ ). La seconde scène (OXVIII = 2.3.3.2.) présente un pileatus dace qui se rend en s'agenouillant devant l'empereur en présence des troupes romaines. La fin de la scène est indiquée par un arbre-barre $(\mathrm{SE18}$ ). La troisième scène (CXIX =2.3.3.3.) nous montre plusieurs combattants daces qui, sous la conduite d'un pileatus, mettent le feu à un site fortifié à l'interieur du quel on peut distinguer quelques grands édifices. La ligoe du coté gauche de la grande fortification de la scène suivante en constitue le tèrme $(\mathrm{SE18} \Rightarrow)$. La quatrième scène $(C X X, C X I=2.3 .3 .4$.) se déroule à l'intérieur d'une très grande forteresse dace sur deux séquences: un group des daces, parmi lesquels beaucoup des pileati se rassemblent autour d'un grand vase (a); un autre group, plus petit, des daces qui s'en meurent ou sont déjà morts. Une muraille diagonale, à l'intérieur de la même forteresse sépare cette scène dramatique de la suivante en marquant aussi la fin de l'épisode (NE18 $\Rightarrow$ ).

Le dernier épisode (2.3.4.) présente les conséquences immédiates de la chute de la capitale et finit aussi par un arbre-barre. La première scène $(C X X I I=2.3 .4 .1$.) présente la retraite des defenseurs de la forteresse de Sarmizegetusa. Le tèrme de cette scène est marqué par un arbre-barre au delà duquel appairessent de nouveau les soldats romains (SV19 $\Rightarrow 10$ 2"). Dans la seconde scène (CXXIII = 2.3.4.2.) l'empereur, accompagné par les troupes reçoit un groupe des comati daces qui se rendent. Une crête rocheuse en diagonale fortement inclinée sépare les scènes (SV19 $\Leftrightarrow 0)$. La troisième scène (CXXIV = 2.3.4.3.) nous présente les troupes faisant des travaux préliminaires dans les environs de Sarmizegetusa: deux soldats transvasent un contenu (a); trois autre portent des

\footnotetext{
${ }^{24}$ Alain Malissard. Op.cit. p.548.
} 
outres remplies à l'intérieur de la forteresse (b); des détails d'architécture et d'aménagments séparent la scène en plusieurs champs. La ligne légérement oblique du coté gauche de la forteresse sépare les scènes. La quatrième scène $(C X X V=2.3 .4 .4$. $)$ se deroule à l'intérieur de la forteresse: en présence des troupes l'empereur harrangue les soldats vainqueurs qui l'acclament. Le coté droite de la citadelle en constitue la limite de la scène et marque la fin de l'épisode et de l'acte (NE20 $\Leftarrow$ ).

3.1.4. Le quatrième acte (2.4.), le dernier, raconte la poursuite des daces et la pacification de la nouvelle province. Sa fin coïncide avec la fin physique de la bande sculptée. Il est possible de distinguer aussi quatre épisodes, composé chacun des plusieurs scènes. Le premier épisode (2.4.1.) présente les premières mésures concernant l'occupation et l'organisation du pays nouvellement conquis. La première scène (CXVVI, CXOVII = 2.4.1.1.) nous montre des travaux de défrichage et de construction des routes et des camps. Elle est séparée de la suivante (CXXVIII, CXXIX, CXOXX = 2.4.1.2.) par un arbre-barre doublé de la ligne oblique du coté gauche d'un camp romain (NV20 $\Rightarrow$ $4 \mathrm{mb})$. Sur le fond de deux camps romains voisins (b) cette seconde scène représente la capitulation d'un group des pileati daces devant l'empereur (a), tandis que des legionnaires amménagent l'intérieur d'un camp (c). Limitée par la rive droite d'un cours d'eau assez grand et rapide, la troisième scène ( $C X X X I, C X X X I I=2.4 .1$.3.) nous présente l'occupation en profondeur du pays dace: les romains passent sur des pontins les cataractes (?) d'une rivière (a); forcent les daces d'abbandoner une fortification faite des rondins de bois. Un arbre-barre sépare cette scène de la suivante(SV20 $\Rightarrow 8 \mathrm{mb}$ ). Celle-ci, la quatrième (COXIV; $C O O X V, C X O X V I=2.4 .1 .4$.) nous depeint le dernier contrattaque des daces/ dans les environs de la citadelle en rondins, maintenant occupée par les romains (a), les daces se rassemblent (b), et assaillisent un camp romain (c); il n'ont pas de succés et se retirent (d). Un arbre-barre sur une roche marque la fin de la scène et aussi de l'épisode (NE21 $\Leftrightarrow 0$ ).

L'épisode suivant (2.4.2.) est celui de la capture des trèsors de Décébale. La première scène (COXVII = 2.4.2.1.) représente de nouveau une harangue. Son tèrme est indiqué par un arbre-barre (NV21 7 1"'). La seconde scène (CXXVIII = 2.4.2.2.) montre des legionnaires romains chargeant sur le dos des mules de la vaisselle en metal. Tous les éxégètes de la Colonne ont été d'accord de mettre cette scène en rapport avec un passage de Dio Cassius ${ }^{29}$, où il s'agit de la capture des trèsors de Décébale. Un arbre-barre et l'apparition des daces indique la fin de la scène (NV21 $\Rightarrow$ ). La troisième scène $(C O O X I X ; O X L=2.4 .2 .3$.) est séparé de la précédente par un arbre-barre et décrit les conséquences de la capture - des combattants daces se suicident ou sont mis à mort par des camarades. La fin de la scène est signalée de nouveau par un arbre-barre (SV21 $\Leftrightarrow 0)$. La quatrième scène (CXLI = 2.4.2.4.) présente la capitulation d'un group des pileati daces. Un arbre-barre sépare cette scène de la suivante et clôt l'épisode (SE21 $=7 \mathrm{mb} 1$ '"').

Le troisème épisode (2.4.3.) est centré sur la mort de Décébale. La première scène (CXLII, CXLIII, CXLV = 2.4.3.1.) représente la poursuite du group régal en fuite par un détachement. de cavalerie romaine. La séparation de cette scène de la suivante est marqué par le commencement de la ligne oblique d'une crête montagneuse dérrière laquelle aparait un autre détachement de cavalerie romaine qui coupe la route des fuyards (SE21 $\Leftrightarrow 0$ ). C'est justement l'intervention de ce deuxièmé détachement qui joue le rôle décisif dans la seconde scène ( $C X L V=2.4 .3 .2$.) où le roi est encérclé et se donne la mort. Un grand arbre-barre indique le tèrme de la scène (NV22 $\Leftrightarrow 0$ ). La troisème scène (CXLVI = 2.4.3.3.) présente la capture des deux adolescents daces (peutêtre après une bref combat) et son fin est indiqué aussi par un grand arbre-barre (SV22 $\Leftrightarrow 0)$. La quatrième scène (CXLVII =

29 Dio Cassius, LxvIII, 14. 
Quelques observations sur le tracee et la partition de la bande a reliefs histories de la Colonne Trajane

2.4.3.4.) est occupée par l'image de la présentation de la tête coupée du roi dace à l'armée romaine rassemblée dans un camp. Un arbre-barre, parallelle au coté droit du camp, indique la fin de la scène et aussi de l'épisode (SV22 $\Rightarrow 20$ ).

Le quatrième épisode (2.4.4.) a comme thème la liquidation des dernières resistences des daces. La première scène (CLXVIII, CXLIX $=2.4 .4 .1$.) représente la capture des deux pileati daces dans un paysage motagneux et sauvage. La fin dela scène est marquée par une haute maison en rondins continuée par un arbre-barre (SE22 $\left.\Rightarrow 13 \mathrm{mb} 1^{\prime \prime \prime}\right)$. La seconde scène (CL; CLI; CLII = 2.4.4.2.) continue avec la capture des deux autres groupes de daces, dont un oppose une certaine résistence. Un grand arbre-barre gémminé indique la fin de la scène (NV23 $\Leftrightarrow 0$ ). La troisième scène (CLII = 2.4.4.3.) nous présente quelques soldats auxiliaires romains qui incendient des maisons fortifiées (?) daces. Une grande crête montagneuse, descendant obliquement de droite à gauche sépare cette scène de la suivante (NV23 $\Rightarrow 13 \mathrm{mb} 2^{\prime \prime \prime}$ ). L a quatrième scène (CLIV, CLV = 2.4.4.4.) présente un group des daces - hommes, femmes et enfants - avec leurs troupeaux excortés par un group des auxilliaires romains ${ }^{30}$. La scène - dont la largeur décroit continuellement - finit en coin, à la fois de la bande sculptée.

Cette nouvelle proposition du découpage de la bande sculptée de la Colonne trajane est assez arride comme démonstration et parraît-il - considéré en soi - être sans objet. Ce nouveau découpage - il faut le dire - n'est que l'essay d'identifier la charpente compositionelle de la bande sculptée et servira comme point de départ et d'appui pour une nouvelle éxégèse de la narration figurée, incluant aussi la nouvelle information obtenue par les fouilles éfféctuées aux cités daces des Carpathes meridionaux comme aussi dans les sites romains de la Dacie et de la Moesie Inferieure. L'auteur est obligé donc de solliciter la patience et l'attention des savants lecteurs pour pouvoir relier les informations contenues dans cette étude avec celles qui apparaitront dans les articles suivantes.

\begin{tabular}{|c|c|c|c|}
\hline \multicolumn{4}{|c|}{$\begin{array}{l}\text { TABLEAU No. 1. DE LA HAUTEUR DES TAMBOURS DE LA COLONNE TRAJANE ET DE LA } \\
\text { VARIATION LOCALE DE LEUR SOUSDIVISION MODULAIRE }\end{array}$} \\
\hline \multicolumn{4}{|r|}{$I^{\prime \prime} 1^{\prime \prime \prime}=0,09240625$} \\
\hline $\begin{array}{l}\text { Tambour } \\
\text { no. }\end{array}$ & $\begin{array}{l}\text { H. en paumes } \\
\text { M:m }\end{array}$ & $\bar{H}$. en mètres & $\begin{array}{l}\text { H. module de base }(=1 / 16: \\
\text { 5p.r. 1 paume 1pouce) }\end{array}$ \\
\hline I & & & \\
\hline II & $6: 111 / 2$ & 1,543184375 & 0,0964490234375 \\
\hline III & $6: 11$ & 1,53394375 & 0,095871484375 \\
\hline IV & $6: 101 / 2$ & 1,524703125 & 0,0952939453125 \\
\hline $\mathrm{V}$ & $6: 9$ & 1,49698125 & 0,093561328125 \\
\hline$\sqrt{\mathrm{I}}$ & $6: 91 / 4$ & 1,5016015625 & 0,09385009765625 \\
\hline VII & $6: 101 / 5$ & 1,51915875 & 0,094947421875 \\
\hline VIII & $6: 8$ & 1,4785 & 0,09240625 \\
\hline$\overline{\mathrm{DX}}$ & $6: 103 / 5$ & 1,52655125 & 0,095409453125 \\
\hline$x$ & $6: 9$ & 1,49698125 & 0,093561328125 \\
\hline$\overline{X I}$ & $6: 11$ & 1,53394375 & 0,095871484375 \\
\hline XII & $6: 103 / 4$ & $1,5 \overline{54071875}$ & 0,0971294921875 \\
\hline XIII & $6: 71 / 3$ & 1,4661791666 & 0,0916361979125 \\
\hline$\overline{\mathrm{XIV}}$ & $6: 81 / 5$ & 1,48219625 & 0,092637265625 \\
\hline$X V$ & $6: 8$ & 1,4785 & 0,09240625 \\
\hline
\end{tabular}

\footnotetext{
${ }^{x}$ La discussion du problème bien controversé si les daces sont déportés hors la nouvelle province romaine de la Dacie ou si, au contraire, ils reviennent d'un réfuge détérminé par la guerre sous la bonne garde de l'armée romaine trouvera sa place propre dans un autre étude.
} 


\begin{tabular}{l|l|l|l|l|}
\hline & XVI & $6: 84 / 5$ & 1,493285 & 0,0933303125 \\
\cline { 2 - 5 } XVII & $6: 9$ & 1,49698125 & 0,093561328125 \\
\hline XVII & $6: 103 / 4$ & 1,5293234375 & 0,09558271484375 \\
\hline
\end{tabular}

Les dimensions ont été prélevées de la planche de Bartoli, exemplaire du M.N.I.R. Bucureşti.

\section{DIMENSIONS FONDAMENTALES}

Circonfèrence tambour $\min .=10,3306 \mathrm{~m} . \sim 36^{\prime}$

Circonfèrence tambour max. $=11,812388 \mathrm{~m} . \sim 40^{\circ}$

\section{CALCUL INVERS}

$40^{\prime}=11,828 \mathrm{~m} . \sim 11,812388 \mathrm{~m}$.

Circonfèrence $/ 8=10^{\prime}=2,957 \mathrm{~m} .=2.1,4785 \mathrm{~m} . \sim 2.1,4765485 \mathrm{~m}$.

D.grand tambour $=3,76 \mathrm{~m}$. R.tambur gros. $=1,88 \mathrm{~m}$.

H.tambour $=1,4785 \mathrm{~m} .=5^{\prime}$

H.tambour $/ 4=0,369 \mathrm{~m}$. Modul de bază $=\mathrm{I}$.tambur $/ 16=0,09240625 \mathrm{~m}$.

H.bande spiralée $=1,18 \mathrm{~m} . \sim 1,107 \mathrm{~m}$.

TABLEAU DES HAUTEURS DES TAMBOURS SUR LA BASE DES DIMENSIONS EN PIEDS (PAUMES) ROMAINS DE LA STAMPE DE PIRANESI

Palmus $=0,073925 ;$ palmus maior $=0,2222 ;$ digitus $=0,0185=0,01848125$

\begin{tabular}{|c|c|c|c|}
\hline No & Dimensions en p.r. & Dimensions en mètres & Module de base \\
\hline 0 & o & o & 0 \\
\hline 1 & $6: 111 / 2$ & $1,3332+0,20329375+0,0092406=1,54573435$ & 0,096608396875 \\
\hline 2 & $6: 11$ & $1,3332+0,20329375=1,53649375$ & 0,096030859375 \\
\hline 3 & $6: 101 / 2$ & $1,3332+0,1848125+0,0092406=1,5272531$ & 0,09545331875 \\
\hline 4 & $6: 9$ & $1,3332+0,16633125=1,49953125$ & 0,093720703125 \\
\hline 5 & $6: 91 / 4$ & $1,3332+0,166332+0,00462032=1,5041515625$ & 0,0940094726562 \\
\hline 6 & $6: 101 / 5$ & $1,3332+0,1848125+0,00369625=1,52170875$ & 0,095106796875 \\
\hline 7 & $6: 8$ & $1,3332+0,14785=1,48105$ & 0,092565625 \\
\hline 8 & $6: 103 / 5$ & $1,3332+0,1848125+0,01108875=1,52910125$ & 0,095568828125 \\
\hline 9 & $6: 9$ & $1,3332+0,16633125=1,49953125$ & 0,093720703125 \\
\hline 10 & $6: 11$ & $1,3332+0,20329375=1,53649375$ & 0,096030859375 \\
\hline 11 & $6: 103 / 4$ & $1,3332+0,1848125+0,013861=1,5318735$ & 0,09574209375 \\
\hline 12 & $6: 71 / 3$ & $1,3332+0,12936875+0,00616042=1,47488959$ & 0,092180599375 \\
\hline 13 & $6: 81 / 5$ & $1,3332+0,14785+0,00369625=1,48474625$ & 0,092796640625 \\
\hline 14 & $6: 8$ & $1,3332+0,14785=1,48105$ & 0,092565625 \\
\hline 15 & $6: 84 / 5$ & $1,3332+0,14785+0,014785=1,495835$ & 0,0934896875 \\
\hline 16 & $6: 9$ & $1,3332+0,16633125=1,49953125$ & 0,093720703125 \\
\hline 17 & $6: 10$ & $1,3332+0,1848125=1,5180125$ & 0,09487578125 \\
\hline
\end{tabular}

TABLEAU NO. 2 - DES PRINCTPALES DIMENSIONS DE LA COLONNE TRAJANE 


\section{TABLEAU NO. 3 - DE LA VARIATION DES CIRCONFÈRENCES DES TAMBOURS ET DE LEUR CORELATION AVEC LE TRACÉE RÉGULATEUR}

Circonfèrence (C) maximale $(\mathrm{CM})=3,68 \times \square=11,5552=39,07744335475$ p.r.

Circonfèrence $(C)$ minimale $(\mathrm{Cm})=3,29 \times \square=10,3306=34,93608386879$ p.r.

Profondeur du relief $(\mathrm{Ar})=0,0328$

Circonfèrence maximale corrigée $=(D M+2 A r) . \square=11,761184=39,77404125803$ p.r. $\sim 40$ p.r.

Circonfèrence minimale corrigée $=(\mathrm{Dm}+2 \mathrm{Ar}) . \square \square 10,536584=35,63268177$

$\square \mathrm{CM} / \mathrm{Cm}$ corrigée $=1,2246=66,26175177545$ d.r.

Ration $\circledast$ per tambour $=0,0765375=41 / 8$ d.r.

Ration @per module $=0,0095671875 \sim 1 / 2$ d.r.

$\mathrm{m}=$ mètres p.r. = pieds romains d.r. = pouces romaines

L.mod. = longueur modulaire $\mathrm{R} / \mathrm{mod}$. = Ration modulaire

\begin{tabular}{|c|c|c|c|c|c|c|c|c|}
\hline Tambour no. & $\mathrm{cm}$ & En p.r; & $\begin{array}{l}\text { Rt en m. } \\
\text { CI-CN }\end{array}$ & R/t en d.r. & $\begin{array}{l}\text { Longueur du } \\
\text { module en mm. }\end{array}$ & yen d.r. & $\begin{array}{l}\text { R/mod. en m. } \\
(L \bmod .1- \\
\text { L. } \bmod . N)\end{array}$ & $\begin{array}{ll}R & \bmod \\
\text { d.r. } & \end{array}$ \\
\hline$I$ & 11,761184 & 40 & b & p & 1470,14805 & 280 & b & $b$ \\
\hline 11 & 11,6846465 & $391 / 2$ & 0,0765375 & $41 / 6$ & 1460,5808125 & 79 & b,0095672375 & $1 / 2$ \\
\hline III & 11,608109 & $391 / 4$ & 0,15307508 & $81 / 4$ & 1451,013625 & $781 / 2$ & 0,019134425 & 1 \\
\hline IV & 11,5315715 & 39 & 0,22961262 & $122 / 5$ & 1441,4464375 & 78 & 0,0287016125 & $11 / 2$ \\
\hline$V$ & 11,455034 & $383 / 4$ & 0,30615 & $161 / 2$ & 1431,87925 & $771 / 2$ & 0,0382688 & 2 \\
\hline VI & 11,3784965 & $381 / 2$ & 0,3826875 & $202 / 3$ & 1422,3120625 & 77 & 0,0478359875 & $22 / 3$ \\
\hline VII & 11,301959 & $381 / 4$ & 0,459225 & $245 / 6$ & 1412,744875 & $761 / 7$ & 0,057403175 & $31 / 8$ \\
\hline VIII & 11,2254215 & 38 & 0,5357625 & 29 & 1403,1776875 & $757 / 8$ & b,0669703625 & $32 / 3$ \\
\hline $\mathrm{Ix}$ & 11,148884 & $375 / 7$ & 0,6123 & $331 / 8$ & 1393,6105 & $752 / 5$ & $b, 07653755$ & $41 / 8$ \\
\hline$x$ & 11,0723465 & $373 / 7$ & 0,6888375 & $371 / 4$ & 1384,0433125 & $746 / 7$ & 0,0861047375 & $42 / 3$ \\
\hline$x$ & 10,995809 & $371 / 6$ & 0,765375 & $412 / 5$ & 1374,476125 & $741 / 3$ & 0,095671925 & $51 / 6$ \\
\hline aI & 10,9192715 & $367 / 8$ & 0,8419125 & $451 / 2$ & 1364,9089375 & $736 / 7$ & 0,1052391125 & $5 / 3$ \\
\hline WII & 10,842734 & $362 / 3$ & 0,91845 & $492 / 3$ & 1355,34175 & $731 / 3$ & 0,1148063 & $61 / 5$ \\
\hline XV & 10,7661965 & $362 / 5$ & 0,9949875 & $535 / 6$ & 1345,7745625 & $725 / 6$ & b,1243734875 & $62 / 3$ \\
\hline$X V$ & 10,689659 & $361 / 6$ & 1,071525 & 58 & 1336,207375 & $721 / 3$ & 0,133940675 & $71 / 4$ \\
\hline$x$ & 10,6131215 & $357 / 8$ & 1,1480625 & $621 / 8$ & 1326,6401875 & $711 / 5$ & p,1435078625 & $73 / 4$ \\
\hline MII & 10,536584 & $352 / 3$ & 1,2246 & $661 / 4$ & 1317,073 & $71 \%$ & b,15307505 & $82 / 7$ \\
\hline
\end{tabular}


TABLEAU No. 4 DES VALEURS DES DISTANCES DES REPÈRES SUR LES AXES CARDINALES DU FÛT A LA SPIRALE, EXPRIMÉES EN mm ET EN MODULES DE BASE

\begin{tabular}{|c|c|c|c|c|c|c|c|c|c|c|c|c|c|c|}
\hline \multicolumn{3}{|c|}{ Tembour } & \multicolumn{3}{|c|}{ Axe NE } & \multicolumn{3}{|c|}{ Axe NV } & \multicolumn{3}{|c|}{ Axe SV } & \multicolumn{3}{|c|}{ Axe SE } \\
\hline No. & M..b: V/16 & Sp. & Reperre & $\mathrm{m}$ & m.b. & Repère & $\mathrm{m}$ & mb. & Repère & $\mathbf{m}$ & mb. & Repetre & $\mathrm{m}$ & m.b. \\
\hline$I$ & 2 & 3 & 4 & 5 & 6 & 7 & 8 & 9 & 10 & $\pi$ & 12 & 13 & 14 & 15 \\
\hline$\underline{S}$ & 96,608397 & 1 & & - & - & $\dot{-}$ & $\div$ & - & - & - & $\cdot$ & $\cdot$ & $\cdot$ & $\cdot$ \\
\hline 0 & 96,608397 & $\mathrm{I}$ & 1.0 & 0 & 0 & - & - & - & - & - & - & - & - & - \\
\hline \multirow[t]{4}{*}{ I } & 96,608397 & $T$ & & & & $\sqrt{2-1}$ & 1270 & 13 & $\mathbb{I I - 1}$ & 960 & 10 & لI-1 & 745 & $7 \overline{4}$ \\
\hline & & 2 & $\pi 2-1=0$ & 1220 & $122 / 3$ & $2-0$ & 0 & 0 & $\pi 2 . I$ & 195 & 2 & $\pi 2-I$ & 425 & $4 \frac{1 / 2}{2}$ \\
\hline & & 2 & $\Downarrow_{I}-2$ & 390 & 4 & 2-I & 0 & 0 & $\cdot$ & $\cdot$ & $\cdot$ & $\cdot$ & - & $\because$ \\
\hline & & 3 & $\pi 3-I$ & 810 & $81 / 2$ & $\pi 3-2=0$ & 1183 & $121 / 4$ & - & - & $\because$ & $\$ \pi-2$ & 1160 & 12 \\
\hline \multirow[t]{2}{*}{$\Pi$} & 96,03086 & 3 & - & - & - & $\cdot$ & 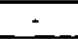 & - & - & - & $\cdot$ & $\pi 3 \cdot \Pi$ & 65 & $2 / 3$ \\
\hline & & 3 & $\rrbracket_{\text {III-3 }}$ & 693 & $71 / 4$ & $\Downarrow_{\Pi \Pi-3}$ & 395 & 4 & $\sqrt{\mathrm{a}-3}$ & 135 & $11 / 2$ & ПI-3 & 0 & 0 \\
\hline \multirow[t]{2}{*}{ III } & 95,453319 & 4 & 14-II & 390 & 4 & $\mathbb{1}_{4-\Pi}$ & 790 & $81 / 4$ & $\pi 4-\square$ & 1100 & $11 \%$ & - & $\overline{-}$ & $\cdot$ \\
\hline & & 4 & $\mathbb{I}_{\text {III-4 }}$ & 1195 & $121 / 2$ & $\sqrt{\text { III-4 }}$ & 780 & $8 \%$ & $\sqrt{\mathrm{m}-4}$ & 542 & $52 / 3$ & $\sqrt{m-4}$ & 270 & 3 \\
\hline \multirow[t]{4}{*}{ IV } & 93.720704 & 5 & 5-III & 0 & 0 & $\Uparrow 5$-II & 400 & 4 & 155-[II & 680 & 7 & - & - & - \\
\hline & & 5 & - & - & $\cdot$ & - & - & - & $\rrbracket_{\text {IV.5 }}$ & 880 & $91 / 2$ & $\sqrt{1 V .5}$ & 615 & $61 / 2$ \\
\hline & & 6 & 16-III & 1195 & $121 / 2$ & 6-IV & 0 & 0 & î6-IV & 390 & 4 & $\pi 6-\mathrm{IV}$ & 620 & $6^{1 / 2}$ \\
\hline & & 6 & - & - & - & $\downarrow$ & 1185 & $122 / 3$ & - & $\cdot$ & $\because$ & - & - & - \\
\hline \multirow[t]{3}{*}{$\mathrm{v}$} & 94,009473 & 7 & $\rrbracket_{\text {IV }-6}$ & 375 & 4 & IV-6 & 0 & 0 & $\downarrow v-6$ & 1180 & $121 / 2$ & $\sqrt{V-6}$ & 904,3 & $91 / 2$ \\
\hline & & 7 & 177-IV & 945 & 10 & 177-rV & 1290 & $13 \%$ & $7-V$ & 0 & 0 & 1ิ7-V & 235,7 & $21 / 2$ \\
\hline & & 8 & WV-7 & 550 & 6 & IV.7 & 290 & 3 & V.7 & 0 & 0 & $\cdot$ & - & $\cdot$ \\
\hline \multirow[t]{2}{*}{ VI } & 95,106797 & $\bar{B}$ & TiB-V & 570 & 6 & $\widehat{\pi}_{\mathrm{B}-\mathrm{V}}$ & 900 & $91 / 2$ & $\pi 8-V$ & 1270 & $131 / 3$ & - & - & $=$ \\
\hline & & 9 & tVI-8 & 1010 & $10^{1 / 2}$ & II $v 1.8$ & 645 & 7 & IVI-B & $32 \overline{2}$ & $31 / 3$ & \VI-8 & 117 & $11 / 4$ \\
\hline \multirow[t]{3}{*}{ VII } & 92,565625 & 9 & $\Uparrow 9 . \mathrm{VI}$ & 210 & $21 / 4$ & T9.VI & 615 & $62 / 3$ & గ9.VI & 920 & $92 / 3$ & î.VI & 1170 & $12 \%$ \\
\hline & & 10 & $=$ & - & - & II VII-9 & 935 & 10 & ฟ Vप-9 & 600 & $61 / 2$ & IV VI-9 & 325 & $31 / 2$ \\
\hline & & 10 & $\cdot$ & - & & $\pi 10-\mathrm{vn}^{-}$ & 340 & $32 / 3$ & $\pi 10 . \mathrm{VI}$ & 580 & $61 / 4$ & $\pi 10-\sqrt{n}$ & 820 & 9 \\
\hline \multirow[t]{2}{*}{ VII } & 95,568829 & I1 & IVII-10 & 220 & $21 / 3$ & $\cdot$ & - & $\cdot$ & IVW-10 & 965 & 10 & $1 \mathrm{VIII-10}$ & 800 & $81 / 3$ \\
\hline & & 11 & $\pi 11-\mathrm{VI}$ & 960 & 10 & - & . & - & Ti1-VII & 260 & $23 / 4$ & ताII-VII & 480 & 5 \\
\hline \multirow[t]{3}{*}{$\overline{\mathbf{x}}$} & 93,720704 & 12 & IVIm-11 & 510 & $51 / 3$ & \vm-11 & 155 & $12 / 3$ & - & - & - & $\Downarrow_{[X-11}$ & 1040 & 11 \\
\hline & & 12 & 112-VIII & 720 & $72 / 3$ & $\pi 12-\mathrm{VII}$ & 935 & 10 & - & - & - & T12-IX & 190 & 2 \\
\hline & & 13 & $\Downarrow \sqrt{X-12}$ & 813 & $82 / 3$ & $\mathbb{W}_{\mathrm{IX}-12}$ & 610 & $61 / 2$ & $\mathbb{I}_{[X-12}$ & 190 & 2 & - & - & - \\
\hline \multirow[t]{2}{*}{$\bar{x}$} & 96,030860 & 13 & $\pi 13-\Gamma X$ & 402 & $41 / 4$ & \$13-IX & 660 & 7 & $\pi 13-\Gamma X$ & 1000 & $102 \sqrt{3}$ & - & - & - \\
\hline & & 14 & - & - & - & $\sqrt{x-13}$ & 1000 & $101 / 3$ & $\downarrow X-13$ & 586 & 6 & $\sqrt{x-13}$ & 350 & $32 / 3$ \\
\hline \multirow[t]{2}{*}{$\mathbf{X I}$} & 95,742094 & 14 & - & - & 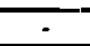 & 1114-X & 270 & 3 & $\pi 14 X$ & 530 & $51 / 2$ & 114-X & 855 & 9 \\
\hline & & 15 & $\sqrt{x-14}$ & 100 & 1 & - & - & - & $\llbracket \mathrm{XI}-14$ & 1050 & 11 & $\sqrt{X-14}$ & 710 & $71 / 2$ \\
\hline XII & 92,180600 & 15 & $\pi 15-X$ & 1080 & $121 / 4$ & - & - & - & đIIS-XI & 100 & 1 & $\pi 15-X 0$ & 395 & 4 \\
\hline & & 16 & $\mathbb{X X I - 1 5}$ & 484 & 5 & $\llbracket \mathrm{XI}-15$ & 255 & $22 / 3$ & - & - & - & $\$$ XII.15 & 1110 & 12 \\
\hline & & 16 & $\mathbb{1} \pi 16-\mathrm{XI}$ & 720 & $7 \mathrm{~K} / \mathrm{s}$ & $\pi 16-X I$ & 990 & $101 / 3$ & - & - & - & 16-XII & 0 & 0 \\
\hline & & 17 & \ХПП-16 & 790 & $81 / 2$ & $\rrbracket \times$ XI-16 & 535 & $53 / 4$ & $\sqrt{\mathrm{XI}-16}$ & $\overline{360}$ & 4 & XII-16 & 0 & 0 \\
\hline XIII & 92,79664 & 17 & its7-XII & 280 & 3 & $\pi 17-X I I$ & 690 & $71 / 2$ & ती17-XII & 800 & $82 / 3$ & (I17.XII & 1130 & 12 \\
\hline & & 18 & - & $\cdot$ & - & $\nabla_{\text {XIII-17 }}$ & 836 & 9 & $\sqrt{X m-17}$ & 670 & $71 / 4$ & $\sqrt{\text { XIII-17 }}$ & 395 & $41 / 4$ \\
\hline xiv & 92,565625 & 18 & - & - & - & 118-XIII & 310 & $31 / 4$ & त18-XIII & 580 & $61 / 4$ & 介18-XIII & 710 & $72 / 3$ \\
\hline & & 19 & $\sqrt{X I I I-18}$ & 150 & $12 / 3$ & - & $\cdot$ & - & $\mathbb{I}_{\mathrm{XTV}-1 \mathrm{~B}}$ & 1005 & $10 \overline{3}$ & $\Downarrow$ XTV-18 & 815 & $8 \%$ \\
\hline$\overline{x y}$ & 93,489688 & 19 & $\pi 19-x \pi I$ & 930 & 10 & $\overline{-}$ & - & - & 19.XIV & 0 & 0 & $\pi 19 \cdot X I V$ & 315 & $31 / 3$ \\
\hline & & 20 & $\sqrt{X T V-19}$ & 565 & 6 & $\rrbracket$ XTV-19 & 280 & 3 & XIV-19 & 0 & 0 & - & $\cdot$ & $\cdot$ \\
\hline & & 20 & $\pi 20-x \mathrm{TV}$ & 535 & $5 \%$ & î20-XIV & 880 & 9 & - & - & - & $\overline{-}$ & - & $\cdot$ \\
\hline & & 21 & $\mathbb{Z X V - 2 0}$ & 1020 & 11 & $\mathbb{X Y}-20$ & 740 & 8 & $\sqrt{x y-20}$ & 220 & $21 / 4$ & - & - & - \\
\hline$\overline{\mathrm{XVI}}$ & 93,720704 & 21 & $\pi 21 \cdot x v$ & 100 & I & $\pi \sqrt{21-X V}$ & 430 & $41 / 2$ & $\pi 21 \cdot x V$ & 940 & 10 & - & - & $\cdot$ \\
\hline & & 22 & - & - & - & $\Downarrow$ XVI-21 & 1370 & $141 / 3$ & $\$$ XVI-21 & 635 & $62 / 3$ & $\llbracket \overline{X V I-21}$ & 300 & 3 \\
\hline & & 22 & - & - & - & $22-X V 1$ & 0 & 0 & $\pi 22-x V I$ & 740 & $7 \%$ & $\pi$ (22-XVI & 1050 & 11 \\
\hline XVII & 94.875782 & 23 & $\overline{X V}-22$ & 0 & $\overline{0}$ & - & - & - & - & $\cdot$ & $\cdot$ & - & - & . \\
\hline XVI & 95,582715 & 23 & XVII-22 & 1500 & $152 / 3$ & $\sqrt{x v a-22}$ & 1340 & 14 & $\sqrt{x V I I-22}$ & 840 & $83 / 4$ & $\rrbracket \times V I I-22$ & 540 & 6 \\
\hline
\end{tabular}


TABLEAU No: 5 - COMPARATIF DES POSITIONS DES CÉSURES SÉPARANT LES ÉPISODES ET LES ACTES PAR RAPPORT AUX AXES CARDINALES DELA COLONNE TRAJANE

\begin{tabular}{|c|c|c|c|c|c|}
\hline Tamb. & Spira & $\mathrm{NE}$ & NV & SV & SE \\
\hline 1 & 1 & & & & $\Leftrightarrow 1.1 .1 .4$ \\
\hline \multirow[t]{2}{*}{2} & 2 & & & $\Leftarrow 1.1 .2 .4$ & \\
\hline & 3 & & & & $\Leftrightarrow 1.1 .3 .4$ \\
\hline 3 & 4 & & & & \\
\hline 4 & 5 & $\Leftarrow 1.1 .4 .4$ & & & $\Leftarrow 1.2 .1 .3$ \\
\hline \multirow[t]{2}{*}{5} & 6 & & & $\Leftarrow 1.2 .2 .4$ & \\
\hline & 7 & $\Leftrightarrow 1.2 .3 .3$ & & & \\
\hline \multirow[t]{2}{*}{6} & 8 & $\Leftrightarrow 1.3 .1 .4$ & & $\Rightarrow 1.3 .2 .4$ & \\
\hline & 9 & & & $\Leftrightarrow 1.3 .3 .4$ & \\
\hline \multirow[t]{2}{*}{8} & 10 & & & & $\Leftarrow 1.3 .4 .4$ \\
\hline & 11 & & $\Leftrightarrow 1.4 .1 .2$ & $\Leftarrow 1.4 .2 .2$ & \\
\hline 9 & 12 & $\Leftarrow 1.4 .3 .2$ & & $\Leftarrow 1.4 .4 .2$ & \\
\hline 10 & 13 & $\Leftarrow 2.1 .1 .2$ & & $\Rightarrow 2.1 .3 .2$ & \\
\hline \multirow[t]{2}{*}{11} & 14 & & $\Leftarrow 2.1 .4 .2$ & & \\
\hline & 15 & $\Rightarrow 2.2 .1 .4$ & & & \\
\hline 12 & 16 & $\Leftarrow 2.2 .2 .4$ & & $\Rightarrow 2.2 .3 .4$ & \\
\hline 13 & 17 & & $\Leftarrow 2.2 .4 .4$ & & $\Leftarrow 2.3 .1 .4$ \\
\hline \multirow[t]{2}{*}{14} & 18 & & $\Rightarrow 2.3 .2 .4$ & & \\
\hline & 19 & & $\Leftarrow 2.3 .3 .4$ & & \\
\hline 15 & 20 & $\Leftarrow 2.3 .4 .4$ & & & \\
\hline 16 & 21 & $\Leftarrow 2.4 .1 .4$ & & $\Rightarrow 2.4 .2 .4$ & \\
\hline \multirow[t]{2}{*}{17} & 22 & & & $\Rightarrow 2.4 .3 .4$ & \\
\hline & 23 & & & & $\Rightarrow 2.4 .4 .4$ \\
\hline
\end{tabular}

1.1.1.1 nombre de la dernjèrre scène d'un episode 1.1.1.1 nombre de la demièrre scène d'un acte 


\section{TABLEAU No. 5 - DES POSTITIONS DES CÉSURES ENTRE LES SCÉNES CALCULÉE EN MODULES DE BASE}

\begin{tabular}{|c|c|c|c|c|c|c|c|c|c|c|c|}
\hline \multirow{2}{*}{$\begin{array}{l}\text { Tam- } \\
\text { bour } \\
\text { No. }\end{array}$} & \multirow{2}{*}{$\begin{array}{l}\text { Spire } \\
\text { no. }\end{array}$} & \multirow{2}{*}{$\begin{array}{c}\text { Longueur du module } \\
\text { de base }\end{array}$} & \multirow{2}{*}{$\begin{array}{l}\text { Scène } \\
\text { no. }\end{array}$} & \multicolumn{4}{|c|}{ Limite gauche } & \multicolumn{3}{|c|}{ Limite droite } & \multirow[b]{2}{*}{$\begin{array}{l}\text { Valeur } \\
\text { modul. }\end{array}$} \\
\hline & & & & $\begin{array}{l}\text { Re- } \\
\text { père }\end{array}$ & Dir. & \begin{tabular}{|c|} 
Valeur \\
métrique \\
\end{tabular} & $\begin{array}{l}\text { Valeur } \\
\text { modulaire }\end{array}$ & Repere & Dir. & $\begin{array}{l}\text { Valeur } \\
\text { métrique }\end{array}$ & \\
\hline \multirow[t]{5}{*}{0} & 1 & 91,884253125 & 1.1 .1 .1 & SE 1 & $\mathbb{}$ & 0 & 0 & NEI & $\Leftrightarrow$ & 0 & 0 \\
\hline & & & 1.1 .1 .2 & NE 1 & $\Leftrightarrow$ & 0 & 0 & NV 1 & $\Leftrightarrow$ & $\mathbf{0}$ & 0 \\
\hline & & & 1.1 .1 .3 & NV 1 & $\Leftrightarrow$ & 0 & 0 & SV 1 & $\Leftarrow$ & $910(?)$ & 10 \\
\hline & & & 1.1 .1 .4 & SV 1 & $\Leftarrow$ & $910 ?$ & 10 & SE 1 & $\Leftrightarrow$ & 0 & 0 \\
\hline & 2 & & 1.1 .2 .1 & SE I & $\Leftrightarrow$ & 0 & 0 & NE 2 & $\Leftrightarrow$ & 0 & 0 \\
\hline \multirow[t]{6}{*}{1} & & 91,28630078125 & 1.1 .2 .2 & NE 2 & $\Leftrightarrow$ & 0 & 0 & NV 2 & $\Leftrightarrow$ & 0 & 0 \\
\hline & & & 1.1 .2 .3 & NV 2 & $\Leftrightarrow$ & 0 & $\overline{0}$ & NV 2 & $\Rightarrow$ & 780 & $81 / 2$ \\
\hline & & & 1.1 .2 .4 & NV 2 & $\Rightarrow$ & 780 & $81 / 2$ & $\bar{s} \mathbf{2}$ & $\Leftarrow$ & 690 & $71 / 2$ \\
\hline & & & 1.1 .3 .1 & SV 2 & $\Leftarrow$ & 690 & $71 / 2$ & SE 2 & $\Leftrightarrow$ & 0 & 0 \\
\hline & 3 & & 1.1 .3 .2 & SE 2 & $\Leftrightarrow$ & $\mathbf{0}$ & $\mathbf{0}$ & $\mathrm{NE} 3$ & $\Leftrightarrow$ & $\mathbf{0}$ & $\begin{array}{ll}0 \\
0\end{array}$ \\
\hline & & & 1.1 .3 .3 & NE 3 & $\Leftrightarrow$ & 0 & 0 & NV 3 & $\Rightarrow$ & 400 & $4^{1 / 2}$ \\
\hline \multirow[t]{5}{*}{2} & & 90,6883515625 & 1.1 .3 .4 & NV 3 & $\Rightarrow$ & 400 & $4 \frac{1 / 2}{2}$ & SE 3 & $\Leftrightarrow$ & $\begin{array}{l}0 \\
0\end{array}$ & 0 \\
\hline & 4 & & 1.1 .4 .1 & $\overline{S E} 3$ & $\Leftrightarrow$ & 0 & $\overline{0}$ & NV 4 & $\Leftrightarrow$ & 0 & 0 \\
\hline & & & 1.1 .4 .2 & NV 4 & $\Leftrightarrow$ & 0 & 0 & SV 4 & $\Leftrightarrow$ & 0 & 0 \\
\hline & & & 1.1 .4 .3 & $\overline{S V} 4$ & $\Leftrightarrow$ & 0 & 0 & SE 4 & $\Leftarrow$ & 365 & 4 \\
\hline & & & 1.1 .4 .4 & SE 4 & $\Leftarrow$ & 365 & 4 & NE 5 & $\Leftarrow$ & 1480 & $161 / 3$ \\
\hline \multirow[t]{6}{*}{3} & 5 & 90,09040234375 & 1.2 .1 .1 & NE 5 & $\Leftarrow$ & 1480 & $161 / 3$ & NV 5 & $E$ & 300 & $3 \mathrm{l} / 3$ \\
\hline & & & 1.2 .1 .2 & NV 5 & $\Leftarrow$ & 300 & $31 / 3$ & SV 5 & $E$ & 1050 & $112 / 3$ \\
\hline & & & 1.2 .1 .3 & SV 5 & $E$ & 1050 & $112 / 3$ & SV 5 & $\Rightarrow$ & 1820 & $201 / 4$ \\
\hline & 6 & & 1.2 .2 .1 & SV 5 & $\Rightarrow$ & 1820 & $201 / 4$ & SE 5 & $\Rightarrow$ & 1230 & $132 / 3$ \\
\hline & & & 1.2 .2 .2 & SE 5 & $\Rightarrow$ & 1230 & $132 / 3$ & NE 6 & $\Rightarrow$ & 900 & 10 \\
\hline & & & 1.2 .2 .3 & NE 6 & $\Rightarrow$ & 900 & 10 & NV 6 & $\Rightarrow$ & 860 & $91 / 2$ \\
\hline \multirow[t]{5}{*}{4} & & 89,492453125 & 1.2 .2 .4 & NV 6 & $\Rightarrow$ & 860 & $91 / 2$ & SV 6 & $\Leftarrow$ & 400 & $41 / 2$ \\
\hline & & & 1.2 .3 .1 & SV 6 & $\Leftarrow$ & 400 & $41 / 2$ & SV 6 & $\Rightarrow$ & 1200 & $13 \frac{1 / 2}{2}$ \\
\hline & 7 & & 1.2 .3 .2 & SE 6 & $\Leftarrow$ & 1200 & $131 / 2$ & SE 6 & $\Rightarrow$ & 1750 & $191 / 2$ \\
\hline & & & 1.2 .3 .3 & SE 6 & $\Rightarrow$ & 1750 & $19 \frac{1 / 2}{2}$ & $\mathrm{NE} 7$ & $\Leftrightarrow$ & 0 & 0 \\
\hline & & & 1.2 .4 .1 & NE 7 & $\Leftrightarrow$ & 0 & 0 & NE 7 & $\Rightarrow$ & 1500 & 17 \\
\hline \multirow[t]{3}{*}{$\cdot$} & & & 1.2 .4 .2 & NE 7 & $\Rightarrow$ & 1500 & 17 & NE 7 & $\Rightarrow$ & 2335 & 26 \\
\hline & & & 1.2 .4 .3 & NV7 & $\Leftarrow$ & 2335 & 26 & NV 7 & $\Rightarrow$ & 1000 & 11 \\
\hline & & & i.2.4.4. & NV7 & $\Rightarrow$ & 1000 & 11 & SV7 & $\Leftrightarrow$ & 0 & 0 \\
\hline \multirow[t]{8}{*}{5} & & 88,89450390625 & 1.3.1.1 & SV 7 & $\Leftrightarrow$ & $\mathbf{0}$ & $\overline{0}$ & SV7 & $\Rightarrow$ & 1420 & 16 \\
\hline & 8 & & 1.3 .1 .2 & SV7 7 & $\Rightarrow$ & 1420 & 16 & SE 7 & $\Leftrightarrow$ & 0 & 0 \\
\hline & & & 1.3 .1 .3 & SE 7 & $\Leftrightarrow$ & 0 & 0 & $\overline{\mathrm{SE} 7}$ & $\Rightarrow$ & 1455 & $161 / 3$ \\
\hline & & & 1.3 .1 .4 & SE 7 & $\Rightarrow$ & 1455 & $161 / 3$ & NE 8 & $\Leftrightarrow$ & 0 & 0 \\
\hline & & & 1.3 .2 .1 & NE 8 & $\Leftrightarrow$ & 0 & 0 & NV 8 & $\Leftarrow$ & 1220 & 13 \\
\hline & & & 1.3 .2 .2 & NV 8 & $\Leftarrow$ & 1220 & 13 & $\overline{N V} 8$ & $\Rightarrow$ & 1070 & 12 \\
\hline & & & 1.3 .2 .3 & SV 8 & $\Leftarrow$ & 1070 & 12 & SV 8 & $\Leftarrow$ & 1210 & $133 / 4$ \\
\hline & & & 1.3 .2 .4 & SV8 & $\Leftarrow$ & 1210 & $132 / 3$ & SV8 & $\Rightarrow$ & 1360 & $151 / 4$ \\
\hline \multirow[t]{6}{*}{6} & 9 & 88,2965546875 & 1.3 .3 .1 & SV 8 & $\Rightarrow$ & 1360 & $151 / 4$ & NE9 9 & $\Leftarrow$ & 1470 & $16^{1 / 2}$ \\
\hline & & & I.3.3.2 & NE 9 & $\Leftarrow$ & 1470 & $161 / 2$ & NE 9 & $\Rightarrow$ & 1250 & 14 \\
\hline & & & 1.3 .3 .3 & NE 9 & $\Rightarrow$ & 1250 & 14 & NV 9 & $\Rightarrow$ & 1470 & $161 / 2$ \\
\hline & & & 1.3 .3 .4 & NV9 & $\Rightarrow$ & 1470 & $161 / 2$ & SV9 & $\Leftrightarrow$ & 0 & 0 \\
\hline & & & 1.3 .4 .1 & SV9 & $\Leftrightarrow$ & 0 & 0 & SE 9 & $\Rightarrow$ & 0 & 0 \\
\hline & 10 & & 1.3 .4 .2 & SE 9 & $\Rightarrow$ & 0 & $\mathbf{0}$ & NE 10 & $\Leftrightarrow$ & 0 & 0 \\
\hline \multirow[t]{4}{*}{7} & & 87,69860546875 & 1.3 .4 .3 & NE 10 & $\Leftrightarrow$ & 0 & $\mathbf{0}$ & SV 10 & $\Leftarrow$ & 440 & 5 \\
\hline & & & 1.3 .4 .4 & SV 10 & $\Leftarrow$ & 440 & 5 & SE 10 & $\Leftarrow$ & 840 & $91 / 2$ \\
\hline & 11 & & 1.4 .1 .1 & SE 10 & $\Leftarrow$ & 840 & $91 / 2$ & SE 10 & $\Rightarrow$ & 1460 & $162 / 3$ \\
\hline & & & 1.4 .1 .2 & SE 10 & $\Rightarrow$ & 1460 & $162 / 3$ & NV II & $\Leftrightarrow$ & 0 & 0 \\
\hline \multirow[t]{3}{*}{8} & & 87,10065625 & 1.4 .2 .1 & NV 11 & $\Leftrightarrow$ & 0 & 0 & NV 11 & $\Rightarrow$ & 520 & 6 \\
\hline & & & 1.4 .2 .2 & NV 11 & $\Rightarrow$ & 520 & 6 & SV 11 & $\Leftarrow$ & 591 & 7 \\
\hline & & & 1.4 .3 .1 & SV 11 & $\varepsilon$ & 591 & 7 & SV 11 & $\Leftrightarrow$ & 0 & $\mathbf{0}$ \\
\hline
\end{tabular}


Quelques observations sur le tracee et la partition de la bande a reliefs histories de la Colonne Trajane

\begin{tabular}{|c|c|c|c|c|c|c|c|c|c|c|c|}
\hline \multirow{2}{*}{$\begin{array}{c}\text { Tam. } \\
\text { No. }\end{array}$} & \multirow{2}{*}{$\begin{array}{l}\text { Spire } \\
\text { No. }\end{array}$} & \multirow[t]{2}{*}{ LMb } & \multirow{2}{*}{$\begin{array}{c}\text { Scène } \\
\text { no. }\end{array}$} & \multicolumn{4}{|c|}{ Limite gauche } & \multicolumn{4}{|c|}{ Limite droite } \\
\hline & & & & Rep. & Dir. & Val.m. & Val.M.b. & Repère & Dir. & val.m. & Val.M.b. \\
\hline \multirow[t]{4}{*}{8} & 12 & 87,10065625 & $1: 4.3 .2$ & SV 11 & $\Leftrightarrow$ & 0 & 0 & NE 12 & $\Leftarrow$ & 600 & 7 \\
\hline & & & 1.4 .4 .1 & NE 12 & $\Leftarrow$ & 600 & 7 & NV 12 & $\Leftarrow$ & 600 & 7 \\
\hline & & & 1.4 .4 .2 & NV 12 & $\Leftarrow$ & 600 & 7 & NV 12 & $\Rightarrow$ & 930 & $102 / 3$ \\
\hline & & & 2.7.1.1 & NV 12 & $\Rightarrow$ & 930 & $102 / 3$ & SE 12 & $\Leftrightarrow$ & 1040 & 12 \\
\hline \multirow[t]{7}{*}{9} & 13 & 87,10065625 & 2.1 .1 .2 & SE 12 & $\Leftarrow$ & 1040 & 12 & SE 12 & $\Rightarrow$ & 1070 & $121 / 3$ \\
\hline & & & 2.1 .2 .1 & SE 12 & $\Rightarrow$ & 1070 & $121 / 3$ & NE 13 & $\Leftarrow$ & 420 & 5 \\
\hline & & & 2.1 .2 .2 & NE 13 & $\Leftarrow$ & 420 & 5 & NV 13 & $\Rightarrow$ & 591,4 & 7 \\
\hline & & & 2.1 .3 .1 & NV 13 & $\Rightarrow$ & 591,4 & 7 & SV 13 & $\Leftarrow$ & 1540 & $173 / 4$ \\
\hline & & & 2.1 .3 .2 & SV 13 & $\Leftarrow$ & 1540 & $173 / 4$ & SV 13 & $\Rightarrow$ & 150 & 2 \\
\hline & & & 2.2 .4 .1 & SV 13 & $\Rightarrow$ & 150 & 2 & SV 13 & $\Rightarrow$ & 2050 & 24 \\
\hline & 14 & & 2.1.4.2 & SV 13 & $\Rightarrow$ & 2050 & 24 & NV 14 & $E$ & 810 & $91 / 2$ \\
\hline \multirow[t]{6}{*}{10} & & 86,50270703125 & 2.2.1.1 & NV 14 & $\Leftarrow$ & 810 & $91 / 2$ & NV 14 & $\Rightarrow$ & 1570 & $181 / 4$ \\
\hline & & & 2.2 .1 .2 & NV 14 & $\Rightarrow$ & 1570 & $181 / 4$ & SV 14 & $\Rightarrow$ & 1160 & $131 / 2$ \\
\hline & 15 & & 2.2 .1 .3 & SV 14 & $\Rightarrow$ & 1160 & $131 / 2$ & SE 14 & $\Leftrightarrow$ & 0 & 0 \\
\hline & & & 2.2.1.4 & SE 14 & $\Leftrightarrow$ & 0 & 0 & NE 15 & $\Rightarrow$ & 1120 & 13 \\
\hline & & & 2.2 .2 .1 & NE 15 & $\Rightarrow$ & 1120 & 13 & NV 15 & $\Rightarrow$ & 750 & $83 / 4$ \\
\hline & & & 2.2 .2 .2 & NV 15 & $\Rightarrow$ & 750 & $83 / 4$ & SV 15 & $\Rightarrow$ & 220 & $21 / 2$ \\
\hline \multirow[t]{7}{*}{11} & & 85,9047578125 & 2.2 .2 .3 & SV 15 & $\Rightarrow$ & 220 & $21 / 2$ & SV 15 & $\Rightarrow$ & 1640 & $191 / 4$ \\
\hline & 16 & & 2.2 .2 .4 & SV 15 & $\Rightarrow$ & 1640 & $191 / 4$ & NE 16 & $\Leftarrow$ & 1200 & 14 \\
\hline & & & 3.2.3.1 & NE 16 & $\Leftarrow$ & 1200 & 14 & NE 16 & $\Rightarrow$ & 660 & $73 / 4$ \\
\hline & & & 2.2.3.2 & NE 16 & $\Rightarrow$ & 660 & $73 / 4$ & NV 16 & $\Leftrightarrow$ & 0 & 0 \\
\hline & & & 2.2 .3 .3 & NV 16 & $\Leftrightarrow$ & 0 & 0 & NV 16 & $\Rightarrow$ & 1600 & $18 \%$ \\
\hline & & & 2.2 .3 .4 & NV 16 & $\Rightarrow$ & 1600 & $183 / 4$ & SV 16 & $\Rightarrow$ & 960 & $111 / 3$ \\
\hline & & & 2.2 .4 .1 & SV 16 & $\Rightarrow$ & 960 & $111 / 3$ & SE 16 & $\Leftrightarrow$ & 0 & 0 \\
\hline \multirow[t]{9}{*}{12} & & 85,30680859375 & 2.2.4.2 & SE 16 & $\Leftrightarrow$ & 0 & 0 & NE 17 & $\Leftarrow$ & 1060 & $12^{1 / 2}$ \\
\hline & 17 & & 2.2 .4 .3 & NE 17 & $\Leftarrow$ & 1060 & $121 / 2$ & NE 17 & $\Leftrightarrow$ & 0 & 0 \\
\hline & & & 2.2.4.4 & NE17 & $\Leftrightarrow$ & 0 & 0 & NE 17 & $\Rightarrow$ & 1500 & $172 / 3$ \\
\hline & & & 2.3.1.1. & NE 17 & $\Rightarrow$ & 1500 & $172 / 3$ & NV 17 & $\Rightarrow$ & 900 & $102 / 3$ \\
\hline & & & 2.3 .1 .2 & NV.17 & $\Rightarrow$ & 900 & $102 / 3$ & sV 17 & $\Leftarrow$ & 600 & 7 \\
\hline & & & 2.3.1.3 & SV 17 & $\varepsilon$ & 600 & 7 & SV 17 & $\Rightarrow$ & 450 & $51 / 3$ \\
\hline & & & 2.3 .1 .4 & SV 17 & $\Rightarrow$ & 450 & $51 / 3$ & SE 17 & $\Leftarrow$ & 750 & 9 \\
\hline & & & 2.3 .2 .1 & SE 17 & $\Leftarrow$ & 750 & 9 & SE 17 & $\Leftrightarrow$ & 0 & 0 \\
\hline & 18 & . & 2.3.2.2 & SE 17 & $\Leftrightarrow$ & 0 & 0 & NE 18 & $\Leftrightarrow$ & 0 & 0 \\
\hline \multirow[t]{7}{*}{13} & & 84,7088593751 & 2.3.2.3 & NE 18 & $\Leftrightarrow$ & 0 & 0 & NV 18 & $\Leftrightarrow$ & 0 & 0 \\
\hline & & & 2.3.2.4 & NV 18 & $\Leftrightarrow$ & 0 & 0 & NV 18 & $\Rightarrow$ & 1260 & 15 \\
\hline & & & 2.3.3.1 & NV 18 & $\Rightarrow$ & 1260 & 15 & SV 18 & $\Rightarrow$ & 400 & $43 / 4$ \\
\hline & & & 2.3 .3 .2 & SV 18 & $\Rightarrow$ & 400 & $43 / 4$ & SE 18 & $\Leftarrow$ & 295 & $31 / 2$ \\
\hline & 19 & & 2.3.3.3 & SE 18 & $\Leftarrow$ & 295 & $31 / 2$ & SE 18 & $\Rightarrow$ & 1100 & 13 \\
\hline & & & 2.3 .3 .4 & SE 18 & $\Rightarrow$ & 1100 & 13 & NV 19 & $\Leftarrow$ & 400 & $43 / 4$ \\
\hline & & & 2.3 .4 .1 & NV 19 & $\Leftarrow$ & 400 & $43 / 4$ & NV 19 & $\Rightarrow$ & 888 & $101 / 2$ \\
\hline \multirow[t]{2}{*}{14} & & 84,11091015625 & 2.3 .4 .2 & SV 19 & $\Rightarrow$ & 888 & $101 / 2$ & SV 19 & $\Leftrightarrow$ & 0 & 0 \\
\hline & & & 2.3 .4 .3 & SV 19 & $\Leftrightarrow$ & 0 & 0 & SE 19 & $\Leftarrow$ & 670 & 8 \\
\hline
\end{tabular}




\begin{tabular}{|c|c|c|c|c|c|c|c|c|c|c|c|}
\hline & 20 & & 2.3 .4 .4 & SE 19 & $=$ & 670 & 8 & NE 20 & $\Leftarrow$ & 1540 & $181 / 2$ \\
\hline & & & 2.4 .1 .1 & NE 20 & $\Leftrightarrow$ & 1540 & $181 / 2$ & NE 20 & $\Rightarrow$ & 340 & 4 \\
\hline & & & 2.4 .1 .2 & NE 20 & $\Rightarrow$ & 340 & 4 & NV 20 & $\Leftrightarrow$ & 0 & 0 \\
\hline & & & 2.4 .1 .3 & NV 20 & $\Leftrightarrow$ & 0 & 0 & SV 20 & $\Rightarrow$ & 660 & 8 \\
\hline & 21 & & 2.4 .1 .4 & SV 20 & $\Rightarrow$ & 660 & 8 & NE 21 & $\Leftrightarrow$ & 0 & 0 \\
\hline 15 & & 83,5129609375 & 2.4 .2 .1 & NE 21 & $\Leftrightarrow$ & 0 & 0 & NV 21 & $\Leftarrow$ & 600 & $71 / 4$ \\
\hline & & & 2.4 .2 .2 & NV 21 & $\Leftrightarrow$ & 600 & $71 / 4$ & NV 21 & $\Rightarrow$ & 600 & $71 / 4$ \\
\hline & & & 2.4 .2 .3 & NV 21 & $\Rightarrow$ & 600 & $71 / 4$ & SV 21 & $\Leftrightarrow$ & 0 & 0 \\
\hline & & & 2.4 .2 .4 & SV 21 & $\Leftrightarrow$ & 0 & 0 & SE 21 & $\Leftrightarrow$ & 600 & $71 / 4$ \\
\hline & 22 & & 2.4 .3 .1 & SE 21 & $\Leftrightarrow$ & 600 & $71 / 4$ & SE 21 & $\Leftrightarrow$ & 0 & 0 \\
\hline 16 & & 82,91501171875 & 2.4 .3 .2 & SE 21 & $\Leftrightarrow$ & 0 & 0 & NV 22 & $\Leftrightarrow$ & 0 & 0 \\
\hline & & & 2.4 .3 .3 & NV 22 & $\Leftrightarrow$ & 0 & 0 & SV 22 & $\Leftrightarrow$ & 0 & 0 \\
\hline & & & 2.4 .3 .4 & SV 22 & $\Leftrightarrow$ & 0 & 0 & SV 22 & $\Rightarrow$ & 1600 & 20 \\
\hline & 23 & & 2.4 .4 .1 & SV 22 & $\Rightarrow$ & 1600 & 20 & SE 22 & $\Rightarrow$ & 1090 & $131 / 4$ \\
\hline & & & 2.4 .4 .2 & SE 22 & $\Rightarrow$ & 1090 & $131 / 4$ & NV 23 & $\Leftrightarrow$ & 0 & 0 \\
\hline & & & 2.4 .4 .3 & NV 23 & $\Leftrightarrow$ & 0 & 0 & NV 23 & $\Rightarrow$ & 1120 & $132 / 3$ \\
\hline & & & 2.4 .4 .4 & NV 23 & $\Rightarrow$ & 1120 & $132 / 3$ & SE 23 & $\Rightarrow$ & 2008 & $241 / 3$ \\
\hline 17 & & 82,3170625 & CHAPIT & SE 23 & $\Rightarrow$ & 2008 & $241 / 3$ & CAPITEL & $\Leftrightarrow$ & 0 & 0 \\
\hline
\end{tabular}


Quelques observations sur le tracee et la partition de la bande a reliefs histories de la colome Trajane

Tableau No 7. Synthétique des dimensions des scènes mesurees sur les reliefs repliques de Bucarest

\begin{tabular}{|c|c|c|c|c|}
\hline No. & Spir. & Axe & Figure & Dimensions \\
\hline 1 & 1 & $\mathrm{NE}$ & & \\
\hline 2 & 1 & $\mathrm{NV}$ & & \\
\hline 3 & 1 & sv & & \\
\hline 4 & 1 & SE & & \\
\hline 5 & 2 & NE & 2 & $\begin{array}{l}2-1=1220=122 / 3 \text { p.r. } \\
2 \rightarrow 0=1220=122 / 3 \\
0-1=0 \\
1.1 .2 .1-\mathrm{NE}=0\end{array}$ \\
\hline 6 & 2 & NV & \begin{tabular}{|r|r|} 
I & $\mid$ \\
1.1 .2 .2 & 1.1 .2 .3 \\
\end{tabular} & $\begin{array}{l}2-1=1270=13 \\
2-I=0 \\
2-F=655 \\
1.1 .2 \cdot 2-N V=0 \\
N V-1.1 .2 .3=780=81 / 2\end{array}$ \\
\hline
\end{tabular}

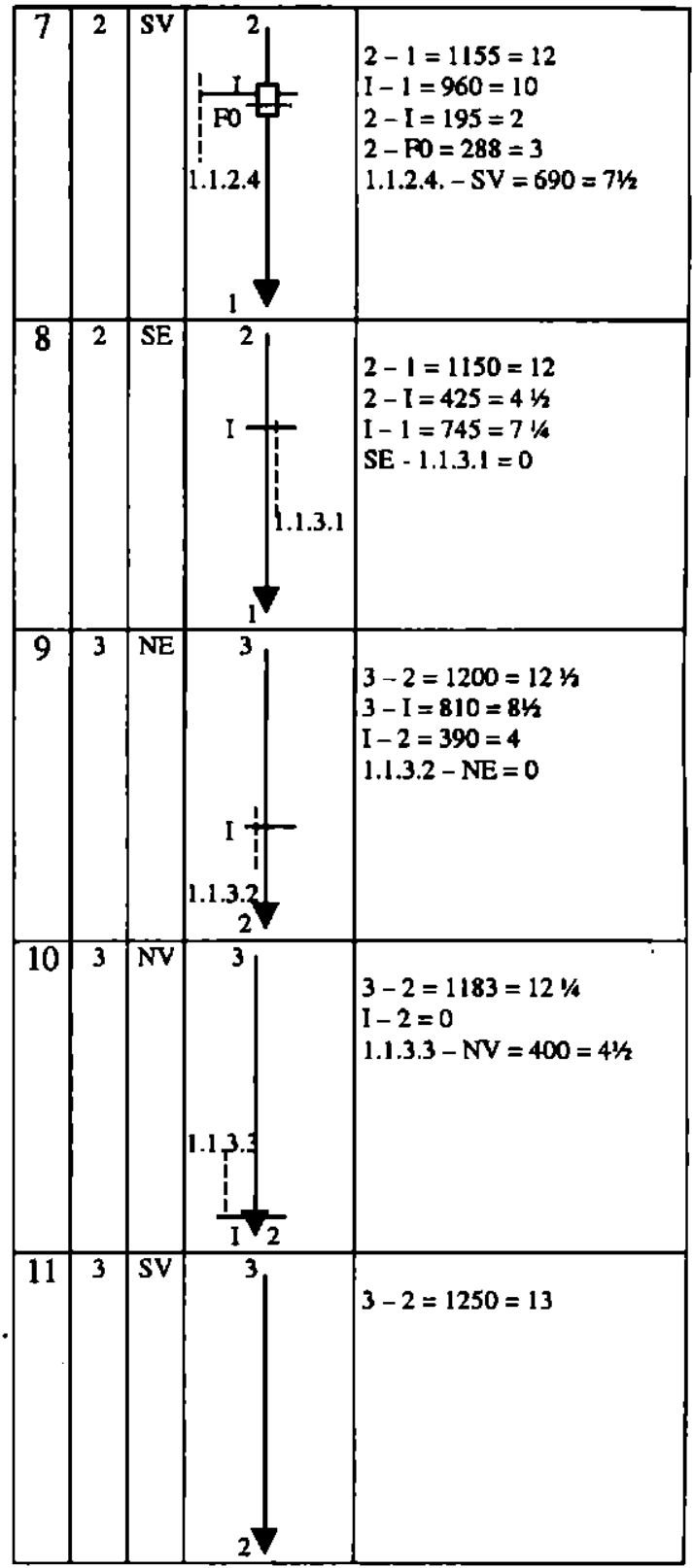




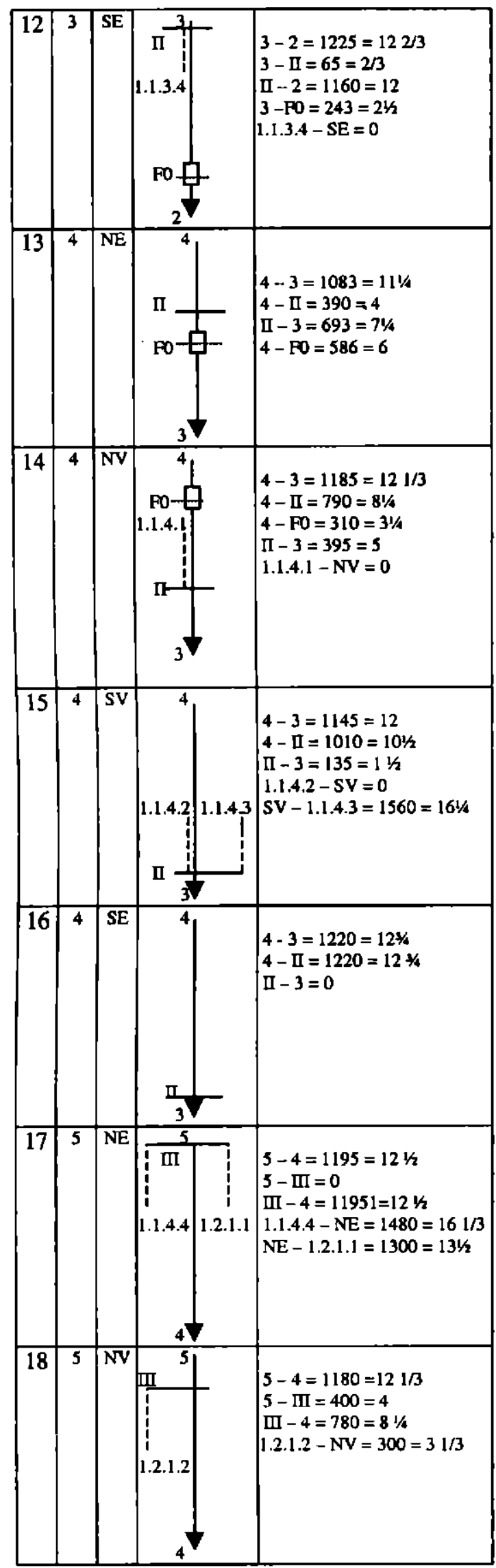

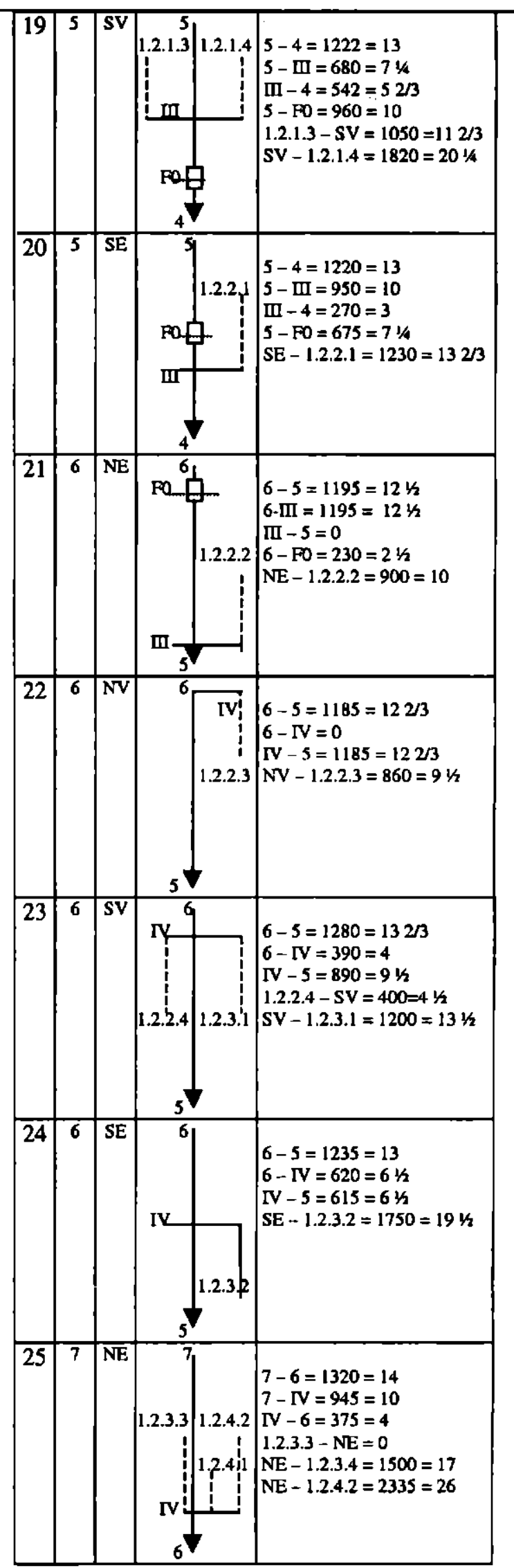


Quelques observations sur le tracee et la partition de la bande a reliefs histories de la Colonne Trajane

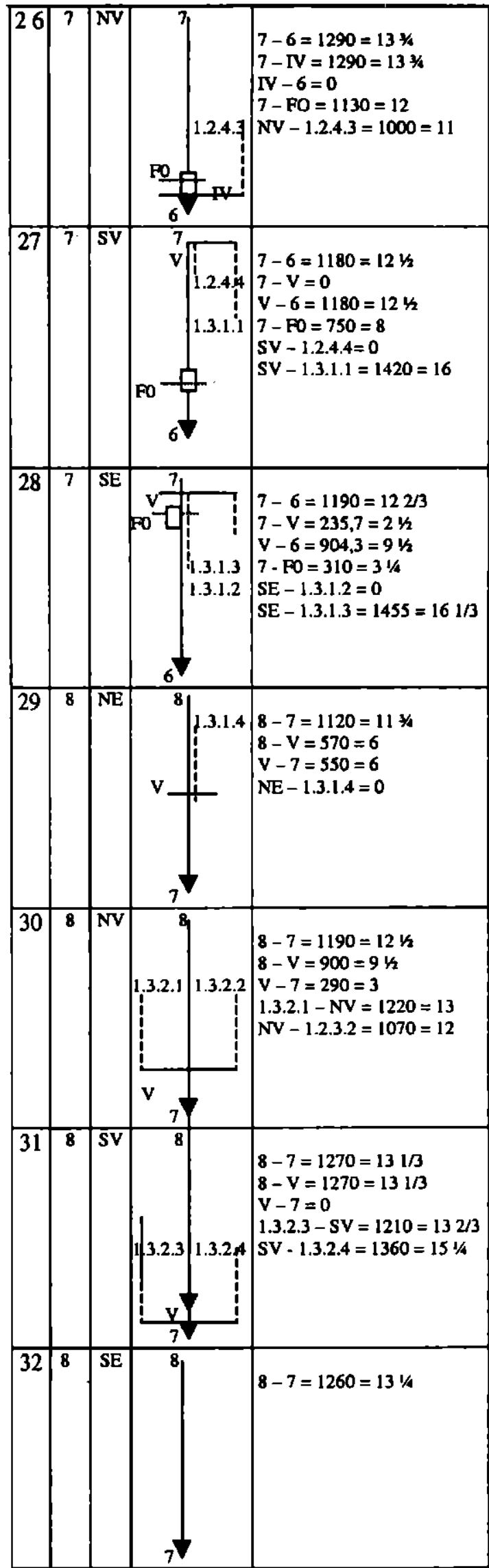

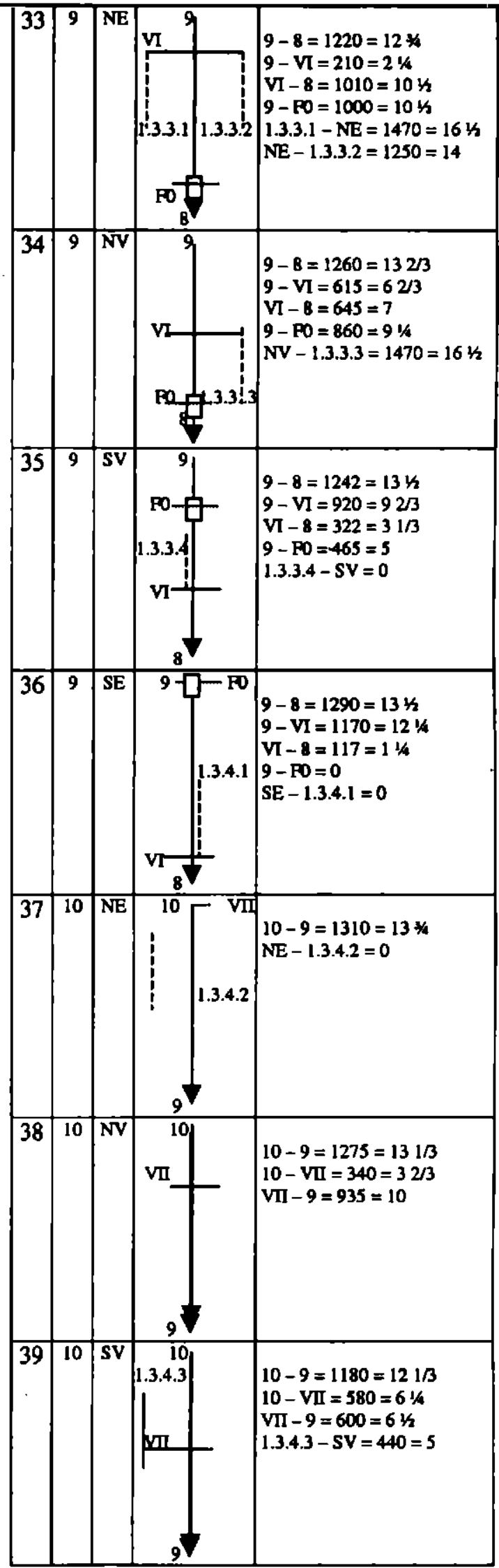




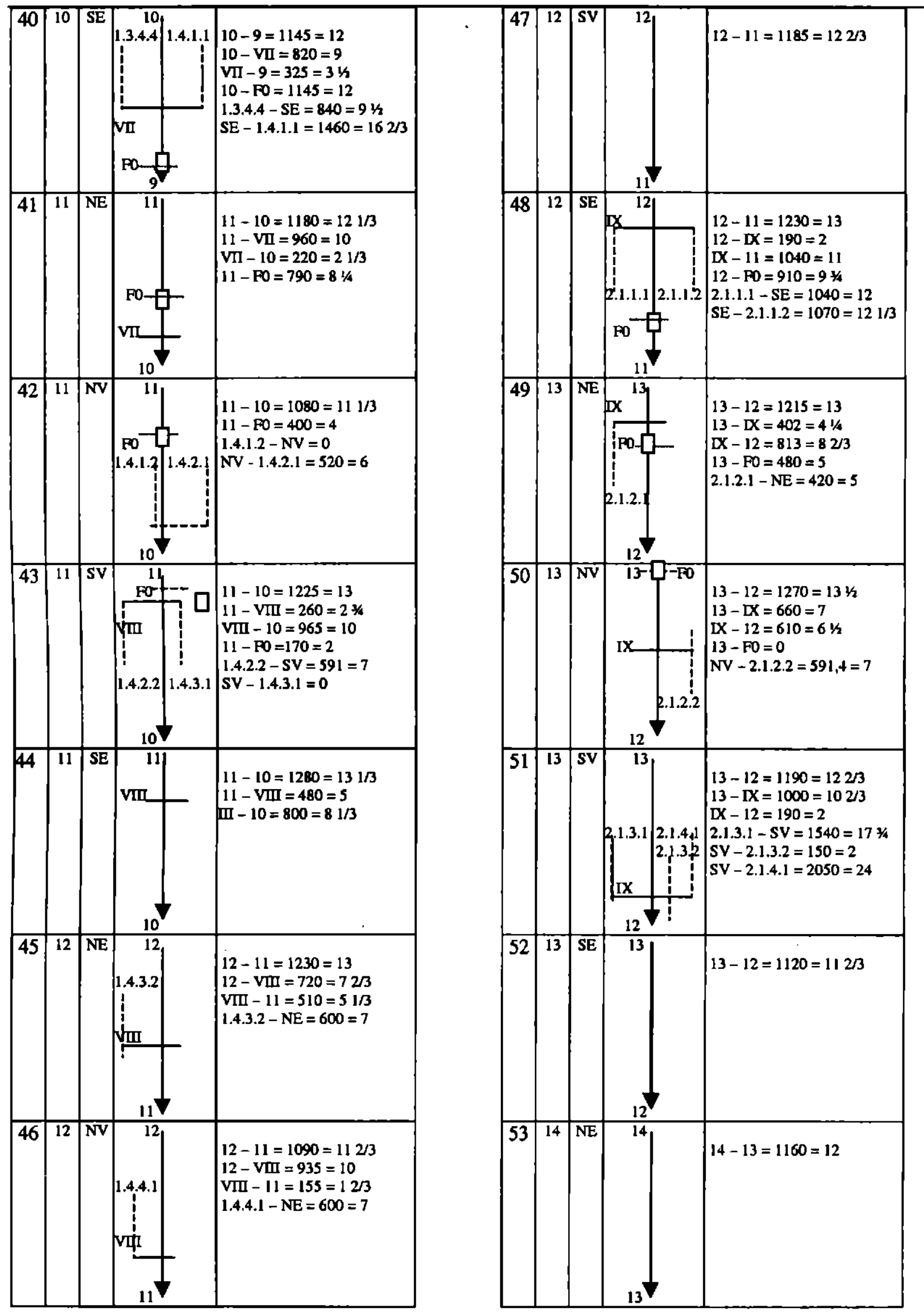


Quelques observations sur le tracee. et la partition de la bande a reliefs histories de la Colonne Trajane

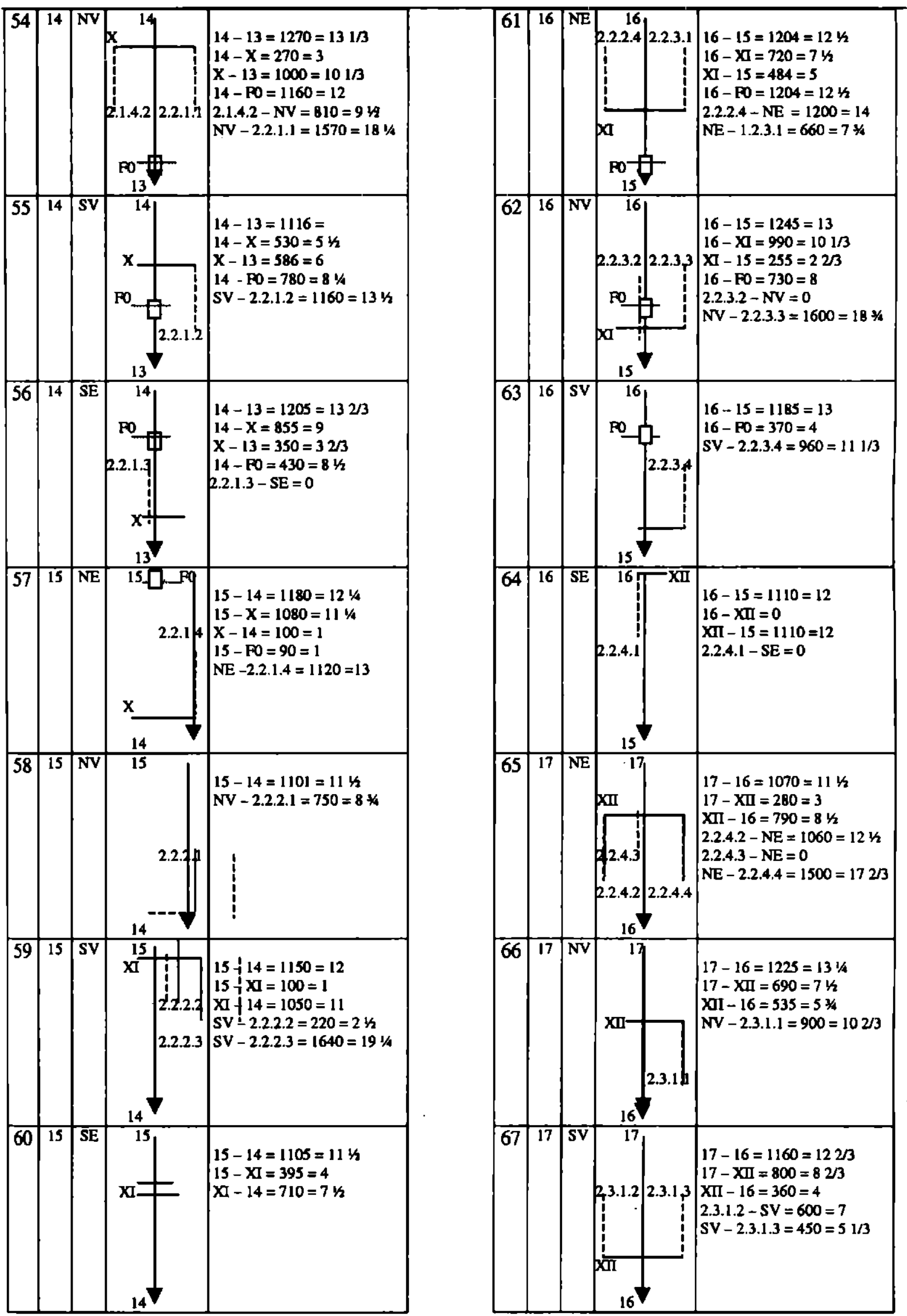




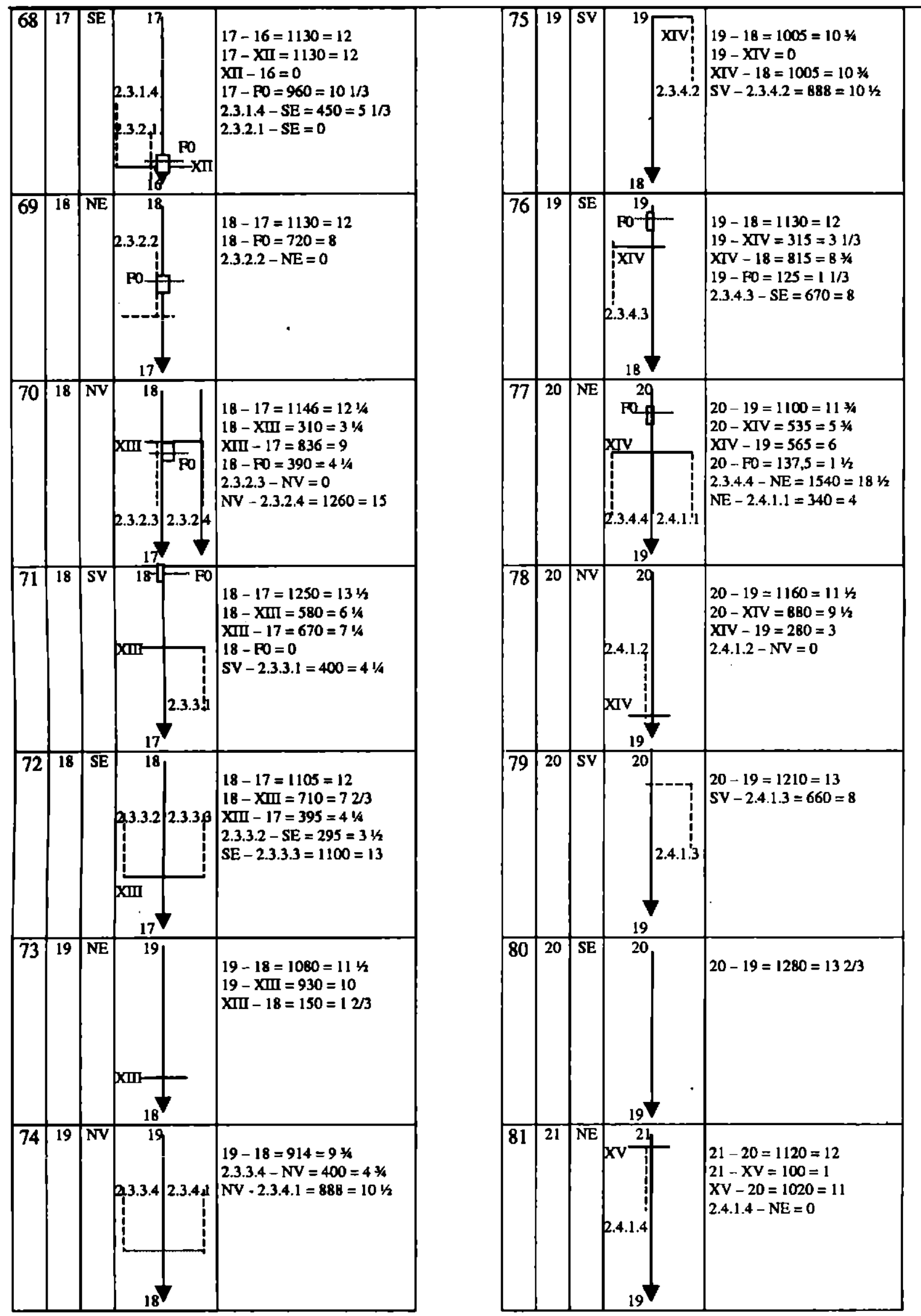


Quelques observations sur le tracee et la partition de la bande a reliefs historles de la Colonne Trajane

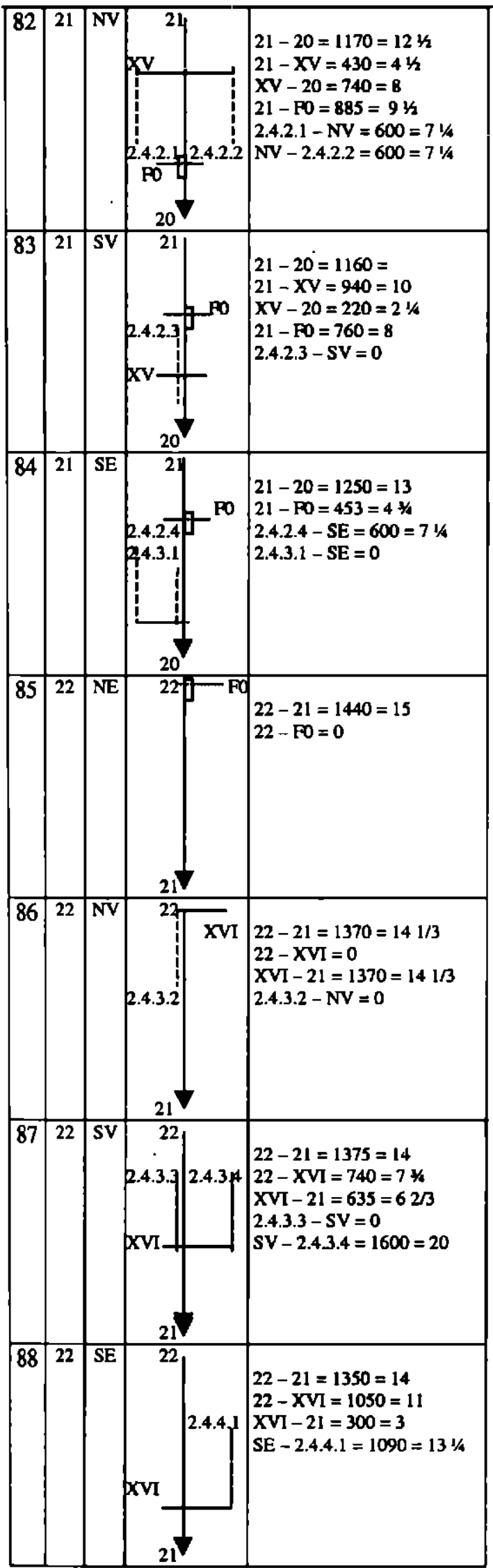
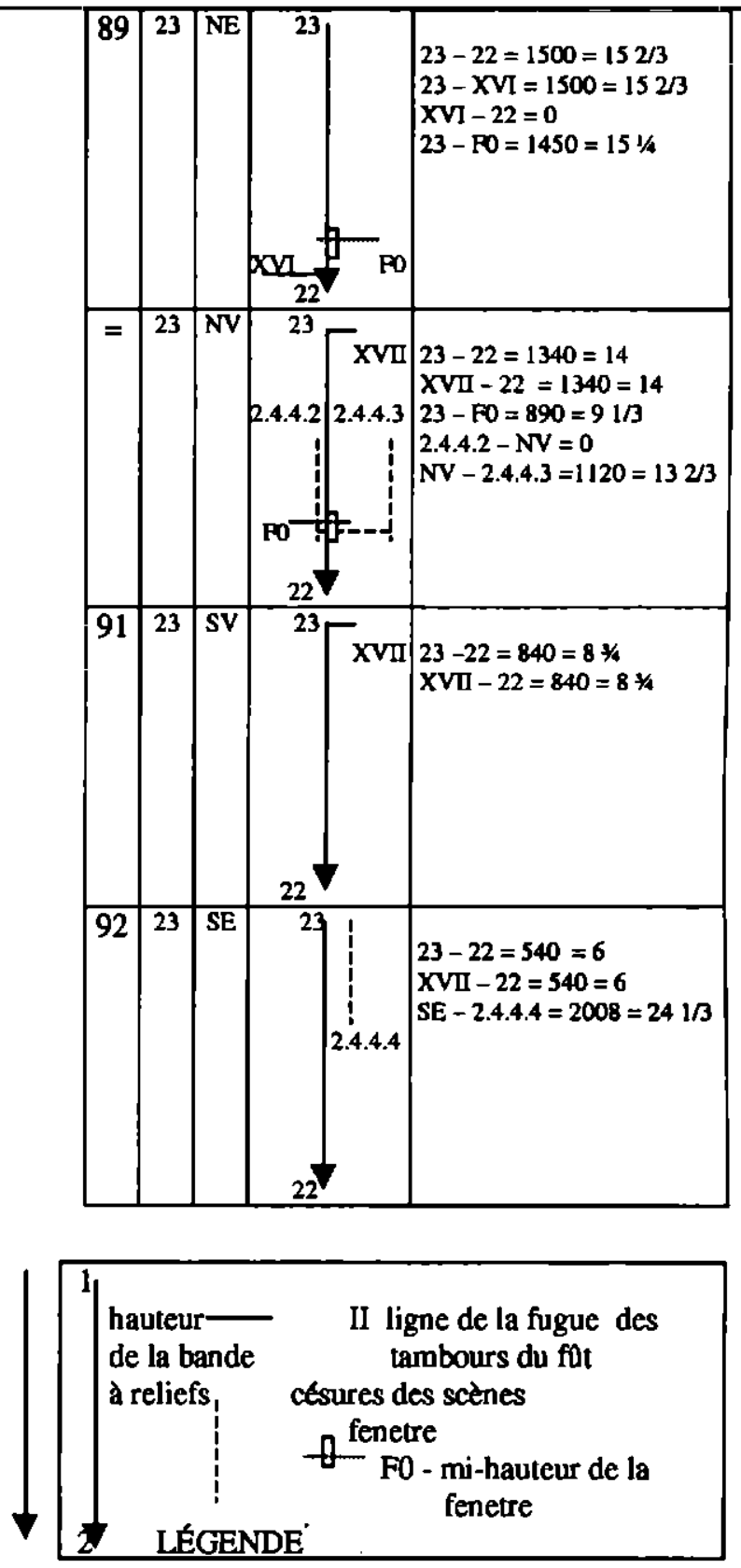

TABLEAU No. 7- RELEVEE DES DIMENSIONS INTÉRIEURES DE LA BANDE SPIRALE A RELIEFS DE LA COLONNE TRAJANE, EN mm ET EN MODULES DE BASE 
TABLEAU No.8 DES GRANDEURS DES SCÈNES, ÉPISODES ET ACTES CALCULÉES EN MODULES DE BASE

$M=$ module $=5$ p.r. de coté

$\mathrm{Mb}=$ module de base $=\mathrm{M} / 16=5 . \mathrm{p} \cdot \mathrm{r} / 16$

Circonference $=8 \mathrm{M}$

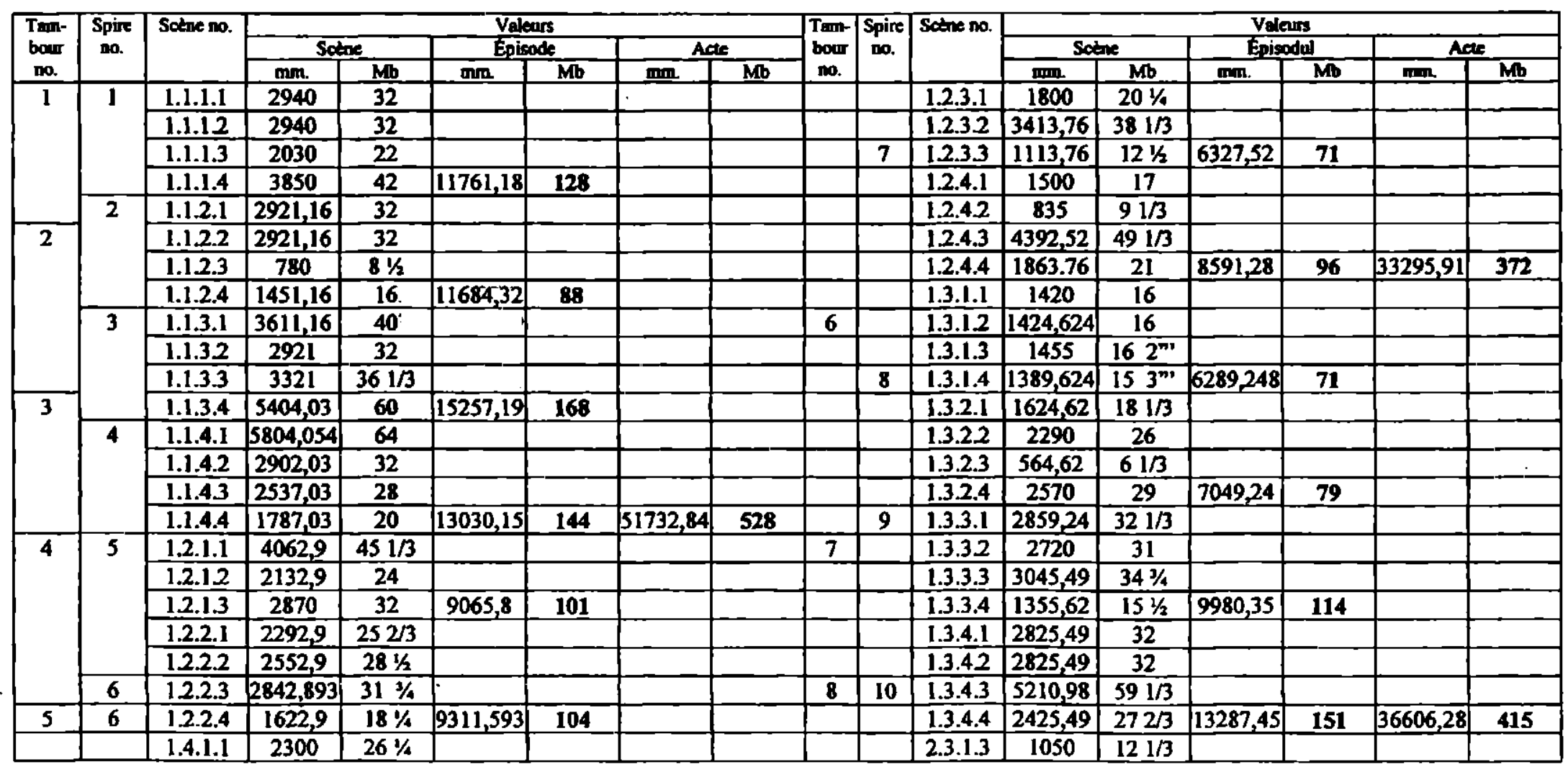




\begin{tabular}{|c|c|c|c|c|c|c|c|c|c|c|c|c|c|c|c|c|c|}
\hline & & 1.4 .12 & 4190,98 & $47 \%$ & 6490 & 74 & & & & & 2.3 .1 .4 & 1510,68 & 18 & 5919,64 & 70 & & \\
\hline \multirow[t]{7}{*}{8} & 11 & 1.42 .1 & 520 & 6 & & & & & & & 2.3 .2 .1 & 750 & 9 & & & & \\
\hline & & 1.4 .2 .2 & 1714 & $192 / 3$ & 2234 & 26 & & & & 18 & 2.3 .2 .2 & 2710,69 & 32 & & & & \\
\hline & & 1.4 .3 .1 & 591 & 7 & & & & & 13 & & 23.23 & 2710,69 & 32 & & & & \\
\hline & 12 & 1.4 .32 & 5050,98 & 58 & 5641,98 & 65 & & & & & 2.3 .2 .4 & 1260 & 15 & 7431,38 & 88 & & \\
\hline & & 1.4 .4 .1 & 2225,49 & $25 \%$ & & & & & & & 23.3 .1 & 1850,69 & 22 & & & & \\
\hline & & 1.4 .4 .2 & 1530 & $172 / 3$ & 3755,49 & 43 & 18121,47 & 208 & & & 2.3 .32 & 2015,69 & $23 \%$ & & & & \\
\hline & & 2.1 .1 .1 & 3642,72 & 42 & & & & & & 19 & 2.33 .3 & \begin{tabular}{|l|}
1395 \\
\end{tabular} & $16 \%$ & & & & \\
\hline \multirow[t]{7}{*}{9} & & 2.1 .12 & 2110 & $24 \%$ & 5752,72 & 66 & & & & & 2.3 .3 .4 & 3921,38 & $462 \sqrt{3}$ & 9182,76 & 109 & & \\
\hline & 13 & 2.12 .1 & 1316,36 & $15 \%$ & & & & & & & 23.4 .1 & 1288 & $15 \%$ & & & & \\
\hline & & 2.122 & 3817,76 & 44 & 5134,12 & 59 & & & 14 & & 2.3 .42 & 1822,69 & $212 / 3$ & & & & \\
\hline & & 2.1 .3 .1 & 674,96 & 8 & & & & & & & 2.3 .4 .3 & 2040,69 & $24 \%$ & & & & \\
\hline & & 2.1 .32 & 1690 & $19 \%$ & 2364,96 & 27 & & & & 20 & 2.3 .4 .4 & 1840,69 & 22 & 6992,07 & 83 & 29525,85 & 350 \\
\hline & & 2.1 .4 .1 & 1900 & 22 & & & & & & & 2.4 .1 .1 & 1880 & $22 \%$ & & & & \\
\hline & 14 & 2.1 .4 .2 & 544426 & 63 & 7344,26 & 85 & 20596,06 & 237 & & & 2.4 .1 .2 & 2351,55 & 28 & & & & \\
\hline \multirow[t]{13}{*}{10} & & 2.2 .1 .1 & 2380 & $271 / 2$ & & & & & & & 2.4 .1 .3 & 3332,42 & 40 & & & & \\
\hline & & 22.1 .2 & 2358,09 & $271 / 4$ & & & & & & 21 & 2.4 .1 .4 & 4684,84 & 56 & 12248,81 & 146 & & \\
\hline & 15 & 2.2 .1 .3 & 1608,09 & $18 \%$ & & & & & 15 & & 2.42 .1 & 3272,42 & 39 & & & & \\
\hline & & 2.2 .1 .4 & 3888,09 & 45 & 10234,27 & 118 & & & & & 2.4 .22 & 1200 & $14 \frac{1}{2}$ & & & & \\
\hline & & 222.1 & 2398,09 & 28 & & & & & & & 2.423 & 2053,28 & $241 / 2$ & & & & \\
\hline & & 22.22 & 2238,09 & 26 & & & & & & & 2.4 .2 .4 & 2053,28 & $241 / 2$ & 8578,98 & 102 & & \\
\hline & & 2223 & I420 & $161 / 2$ & & & & & & 22 & $2.4 . \overline{3.1}$ & 600 & 714 & & & & \\
\hline & 16 & 2.22 .4 & 2696,18 & $311 / 3$ & 8752,36 & 102 & & & 16 & & 2.4 .32 & 530656 & 64 & & & & \\
\hline & & 2.2 .3 .1 & 1860 & $21 \mathrm{~K} / 2$ & & & & & & & 2.4 .3 .3 & 2634,15 & 32 & & & & \\
\hline & & 2232 & 2069,82 & $24 \%$ & & & & & & 4 & 2.4 .3 .4 & 1600 & 20 & 10140,71 & 123 & & \\
\hline & & 22.3 .3 & \begin{tabular}{|l|}
1600 \\
\end{tabular} & $183 / 4$ & & & & & & 23 & 2.4.4.1 & 2124,15 & 26 & & & & \\
\hline & & 223.4 & 2089,82 & $24 \%$ & 7628,64 & 89 & & & & & 2.4 .42 & 4178,3 & $50 \%$ & & & & \\
\hline & & 2.2 .4 .1 & 1769,82 & $20 \%$ & & & & & & & 2.4 .4 .3 & 1120 & $132 / 3$ & & & & \\
\hline \multirow[t]{5}{*}{12} & & 22.42 & 1669,82 & $19 / 2$ & & & & & 17 & & 2.4 .4 .4 & 6156,3 & 75 & 13578,75 & 165 & 44547,25 & 536 \\
\hline & 17 & 22.43 & 1060 & $12 \%$ & & & & & & & & & & & & & \\
\hline & & 2.2 .4 .4 & 1500 & $172 / 3$ & 5999,64 & 87 & 32614,91 & 396 & & & & & & & & & \\
\hline & & 2.3.1.I & 2129,82 & 29 & & & & & & & & & & & & & \\
\hline & & 2.3 .12 & 1229,82 & $141 / 2$. & & & & & & & & & & & & & \\
\hline
\end{tabular}


TABLEAU NO. 9 - COMPARATIF DES DIMENSIONS DES ACTES ET ÉPISODES EXPRIMÉS EN MODULES DE BASE

\begin{tabular}{|c|c|c|c|c|c|}
\hline Acte & Dimension & Eplsode 1 & Episode 2 & Episode 3 & Episode 4 \\
\hline 1.1 & 528 & 128 & 88 & 168 & 144 \\
\hline 1.2 & 372 & 101 & 104 & 71 & 96 \\
\hline 1.3 & 415 & 71 & 79 & 114 & 151 \\
\hline 1.4 & 208 & 74 & 26 & 65 & 43 \\
\hline 2.1 & 237 & 66 & 59 & 27 & 85 \\
\hline 2.2 & 396 & 118 & 102 & 89 & 87 \\
\hline 2.3 & 350 & 70 & 88 & 109 & 83 \\
\hline 2.4 & 536 & 146 & 102 & 123 & 165 \\
\hline
\end{tabular}

Premlère guerre $=1523 \mathrm{mb}$

Seconde guerre $=1519 \mathrm{mb}$

Totale

$=3042 \mathrm{mb}$ 


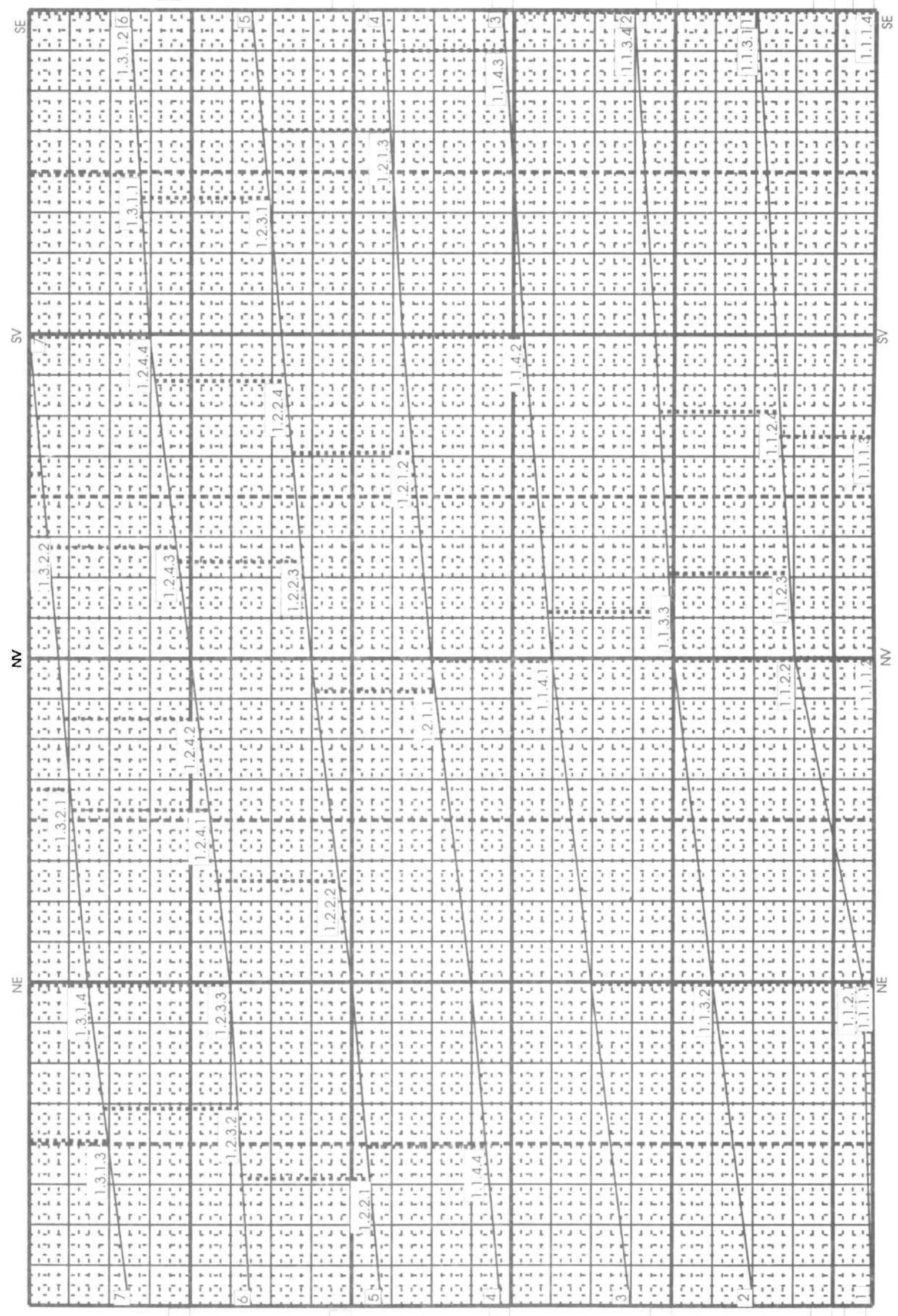




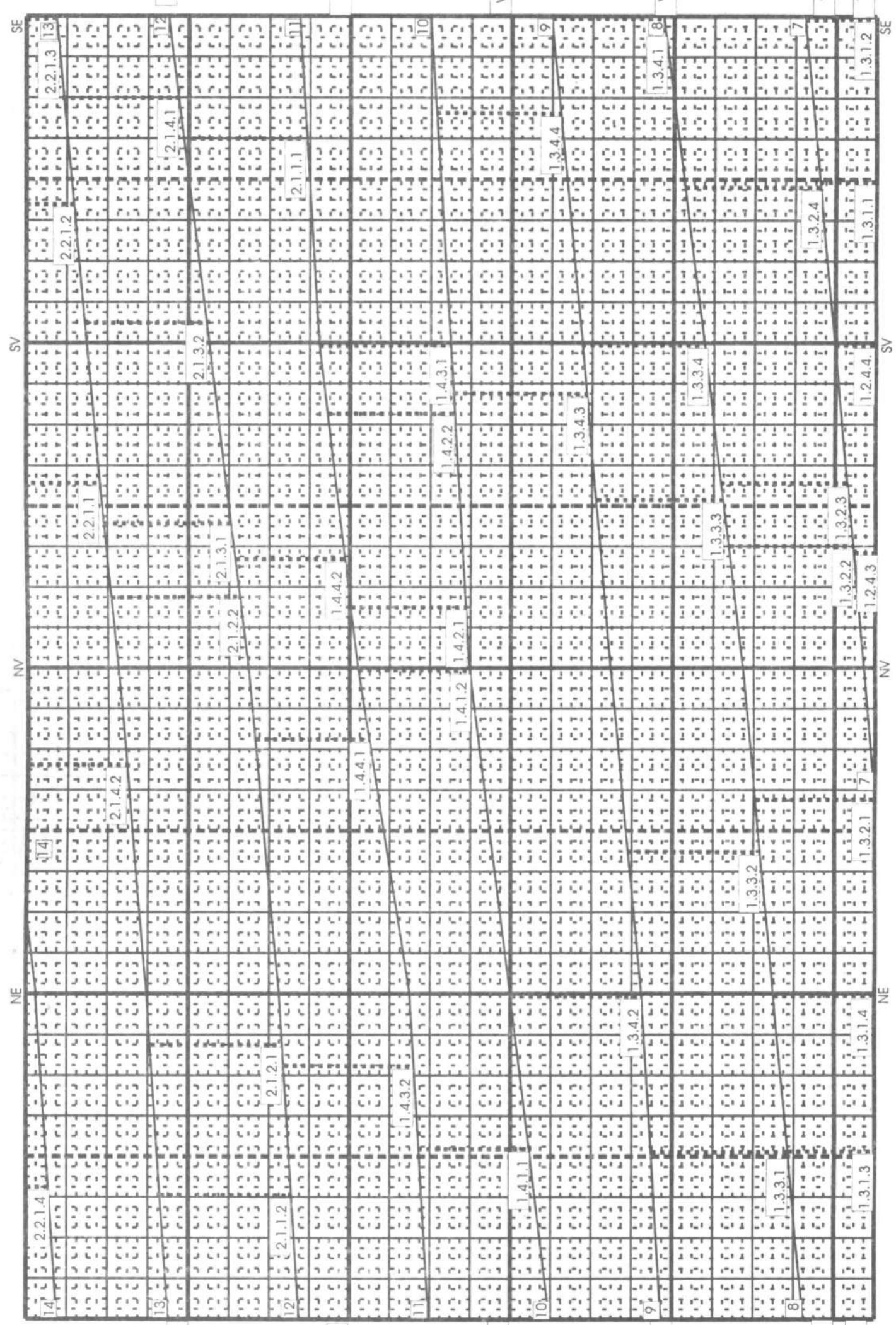




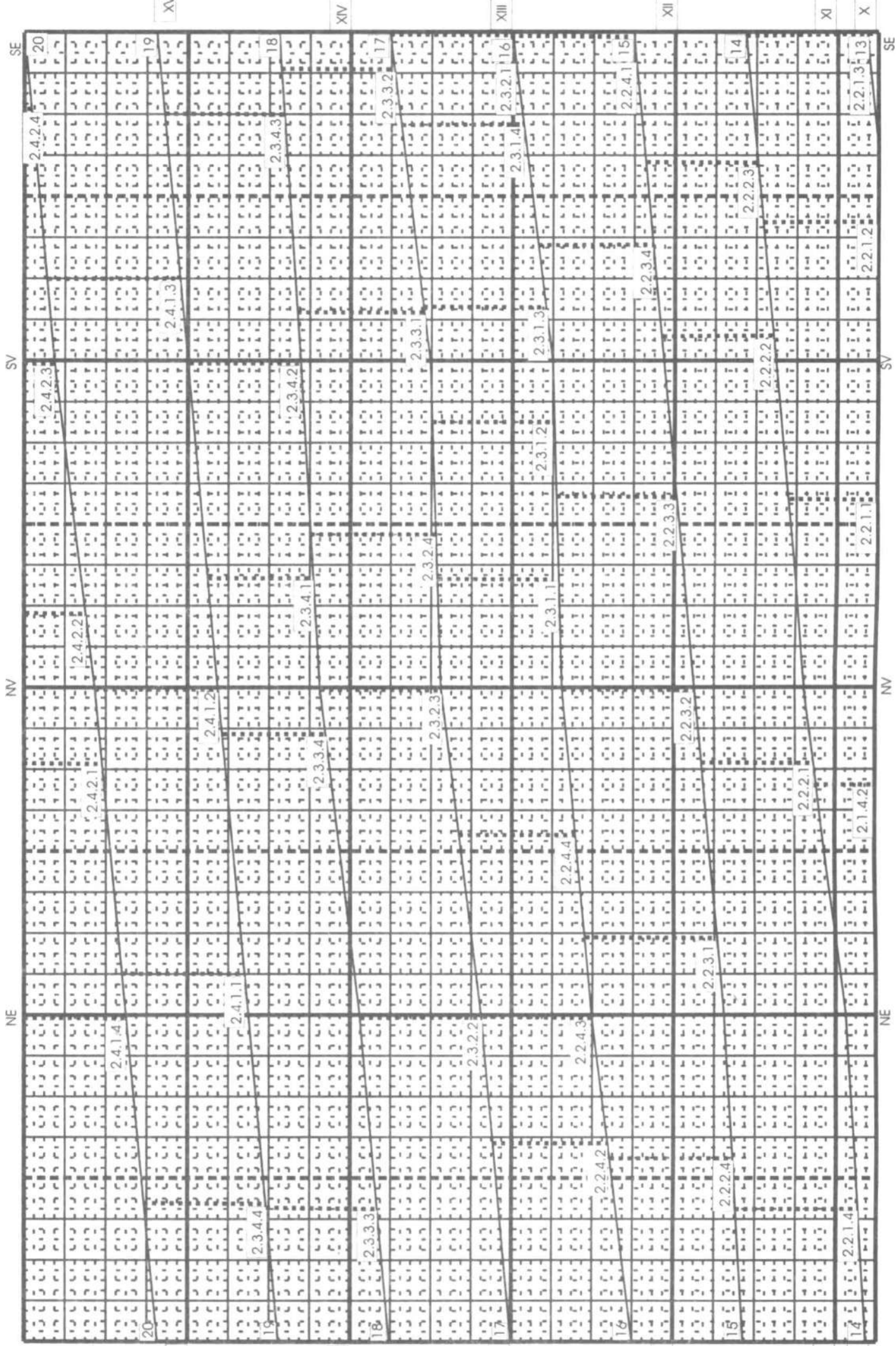


LE TRACÉ DE LA BANDE SPIRALE DU FUT DE LAI COLONNEI TRAJANNE AVEC LE MARQUAGE DES CESURES ENTRE LES SCENES

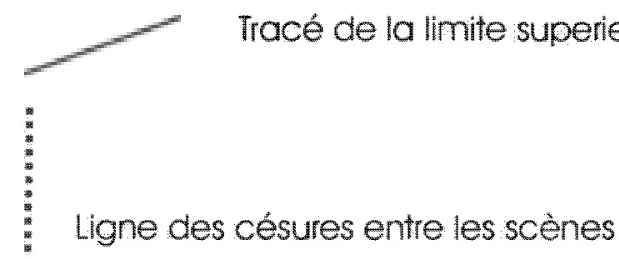

1.1.1.1 Nombre d'ordre des scènesr selon la variante d'ordonnance Radu Florescu lles scènes sont numeroté en système binaire: 1. Nombre de la querre
1.1. Nombre de l'acte
1.1.1. Nombre de l'épisode
1.1.1.1 Nombre de la scène]

xil 